\title{
Bayesian case-deletion model complexity and information criterion
}

\author{
Hongtu Zhu ${ }^{\dagger}$, Joseph G. Ibrahim ${ }^{\dagger, *}$, And Qingxia Chen ${ }^{\ddagger}$
}

We establish a connection between Bayesian case influence measures for assessing the influence of individual observations and Bayesian predictive methods for evaluating the predictive performance of a model and comparing different models fit to the same dataset. Based on such a connection, we formally propose a new set of Bayesian case-deletion model complexity (BCMC) measures for quantifying the effective number of parameters in a given statistical model and its properties in linear models are explored. Adding certain functions of BCMC to a conditional deviance function leads to a Bayesian case-deletion information criterion (BCIC) for comparing models. We systematically investigate some properties of BCIC and its connections with other information criteria, such as the Deviance Information Criterion (DIC). We illustrate the proposed methodology for the linear mixed model with simulations and a real data example.

Keywords AND PHRASES: Bayesian, Case influence measures, Cross validation, Information criterion, Markov chain Monte Carlo, Model complexity.

\section{INTRODUCTION}

The aim of this paper is to establish a formal connection between Bayesian case influence measures for assessing the influence of individual observations on a model and Bayesian predictive methods for choosing an appropriate dimension of a model and selecting the best model for a given dataset. In Bayesian analysis, such statistical measures are very important and highly relevant in any formal statistical analysis, but their formal connections have not been fully explored. We systematically examine the properties of these measures and establish such connections in this paper.

Bayesian case influence measures are typically developed for the purpose of assessing the influence of individual observations (or generally, a set of observations), but they also

* Corresponding author.

${ }^{\dagger}$ Dr. Zhu and Dr. Ibrahim's work was partially supported by RR025747-01, GM70335, CA74015, P01CA142538-01, MH086633, and EB005149-01 from the National Institutes of Health; as well as SES1357666 and DMS-1407655 from the National Science Foundation.

$\ddagger^{\ddagger}$ Dr. Chen’s work was partially supported by 1R21HL097334 and UL1 RR024975-01 from the National Institutes of Health. provide a measure of the importance of each observation in the analysis for assessing model fit $[33,9,25,5,7,12,11]$. See $[42,43]$ for a comprehensive review of various Bayesian case influence measures and their properties. In particular, single case influence measures have been widely used for various specific statistical models including generalized linear models, time series models, survival models, and statistical models with missing data $[18,29,20,14,12,28,43]$. The influence of individual observations are often assessed either on the posterior distributions or the predictive distributions through case deletion. The two most popular Bayesian case influence measures are the Kullback-Leibler (KL) divergence [11] and the conditional predictive ordinate (CPO) $[14,12]$.

Bayesian predictive methods are developed to evaluate the predictive performance of a given model and to select a single model with the best predictive performance from a set of candidate models. For instance, many researchers have been interested in Bayesian model assessment tools based on criterion-based methods, such as the $L$-measure $[17,23,15,16,8]$. See [39] and [4] for an overview of recent progress in cross-validation procedures and Bayesian predictive methods for model assessment, selection, and comparison. The main challenge is to estimate predictive model accuracy by correcting for the bias inherent in the double use of the data for both fitting and prediction. Crossvalidation $(\mathrm{CV})$ is a natural way of estimating out-of-sample prediction error $[12,41]$. However, since cross-validation requires repeated model fits, it is computationally intensive, and hence, information criteria are commonly sought as alternative measures. Such information criteria include the Akaiki Information Criterion (AIC) [1], the Takeuchi Information Criterion (TIC) [35, 22], the Bayesian Information Criterion (BIC) [31, 24, 21], the Deviance Information Criterion (DIC) [32], and the Bayesian Predictive Information Criterion (BPIC) [2], among many others. All these information criteria incorporate different complexity terms for model choice and can be viewed as approximations to different versions of cross-validation [34, 33].

Despite the extensive literature on Bayesian diagnostic measures and Bayesian predictive methods, very little has been done on systematically examining their connections in general parametric models. Based on the connections explored here, we also develop Bayesian case-deletion model complexity (BCMC) measures for quantifying the effective number of parameters in a given statistical model and 
a Bayesian case-deletion information criterion (BCIC) for comparing different models. We calculate BCMC and BCIC in two theoretical examples involving linear models and linear mixed models. We show that BCMC can be regarded as a measure of model complexity, and show its asymptotic equivalence to the effective number of parameters in various information criteria. We systematically investigate the connection of BCIC with cross-validation methods and other information criteria, such as TIC and DIC. When the number of observations in each set, denoted as $N_{S}$, is small, we systematically derive their asymptotic approximations, which facilitate their computation and establish their asymptotic equivalence.

The rest of this paper is organized as follows. In Section 2, we review Bayesian case influence measures and Bayesian predictive methods. We propose $\mathrm{BCMC}$ for measuring model complexity and BCIC for comparing different models. We also systematically establish the connections between our two new measures including BCMC and BCIC as well as many existing model complexity measures and information criteria. In Section 3, we illustrate the proposed methodology using both simulations and a real dataset involving the Yale infant growth data for the linear mixed model. We conclude the paper with some discussion in Section 4.

\section{METHODS}

\subsection{Bayesian case influence measures}

We consider a probability density function for an $N \times 1$ vector $\boldsymbol{Y}^{T}=\left(\boldsymbol{Y}_{1}^{T}, \ldots, \boldsymbol{Y}_{n}^{T}\right)$, denoted by $p(\boldsymbol{Y} \mid \boldsymbol{\theta})$, where $\boldsymbol{\theta}=$ $\left(\theta_{1}, \ldots, \theta_{p}\right)^{T}$ is a $p \times 1$ vector in an open subset $\Theta$ of $R^{p}$, $\boldsymbol{Y}_{i}=\left(y_{i 1}, \ldots, y_{i m_{i}}\right)^{T}$, and $N=\sum_{i=1}^{n} m_{i}$. Letting $p(\boldsymbol{\theta})$ be the prior distribution of $\boldsymbol{\theta}$, the posterior distribution for the full data $\boldsymbol{Y}$ is given by $p(\boldsymbol{\theta} \mid \boldsymbol{Y}) \propto p(\boldsymbol{Y} \mid \boldsymbol{\theta}) p(\boldsymbol{\theta})$. Moreover, the dimension of $\boldsymbol{Y}_{i}$ (or $m_{i}$ ), such as the number of repeated measures in each cluster for longitudinal studies, may vary across all $i$.

Bayesian case influence measures are primarily used to assess the influence of deleting an $N_{S} \times 1$ vector of observations, denoted by $S$, on posterior inferences regarding $\boldsymbol{\theta}$. We use a subscript ' $[\mathrm{S}]$ ' to denote the relevant quantity with all observations in $S$ deleted. For example, if $S=\{i\}$, then $\boldsymbol{Y}_{[S]}$ is the corresponding observed data with all of $\boldsymbol{Y}_{i}$ deleted, whereas for $S=\left\{i_{1}, i_{2}\right\}, \boldsymbol{Y}_{[S]}$ is the corresponding observed data with $\boldsymbol{Y}_{i_{1}}$ and $\boldsymbol{Y}_{i_{2}}$ deleted. Moreover, we may set $S=\left\{i_{1}, \ldots, i_{k}\right\}$ and $S=\left\{\left(i_{1}, j_{1}\right), \ldots,\left(i_{k}, j_{k}\right)\right\}$ to allow more complicated case deletions. We use $\boldsymbol{Y}_{S}$ and $\boldsymbol{Y}_{[S]}$ to represent a subsample of $\boldsymbol{Y}$ consisting of all the observations in $S$ and a subsample of $\boldsymbol{Y}$ with all observations in $S\left(\boldsymbol{Y}_{S}\right)$ deleted, respectively. We also calculate $p\left(\boldsymbol{\theta} \mid \boldsymbol{Y}_{[S]}\right) \propto p\left(\boldsymbol{Y}_{[S]} \mid \boldsymbol{\theta}\right) p(\boldsymbol{\theta})$ as the posterior distribution of $\boldsymbol{\theta}$ given $\boldsymbol{Y}_{[S]}$, where $p\left(\boldsymbol{Y}_{[S]} \mid \boldsymbol{\theta}\right)=p(\boldsymbol{Y} \mid \boldsymbol{\theta}) / p\left(\boldsymbol{Y}_{S} \mid \boldsymbol{\theta}\right)$.

Following [43], we briefly introduce three types of Bayesian case influence measures based on case deletion. First, we consider the $\phi$-influence of $\boldsymbol{Y}_{[S]}$, denoted by
$\mathrm{D}_{\phi}(S)$, as a measure of the distance (discrepancy) between $p\left(\boldsymbol{\theta} \mid \boldsymbol{Y}_{[S]}\right)$ and $p(\boldsymbol{\theta} \mid \boldsymbol{Y})$. Letting $R_{[S]}(\boldsymbol{\theta})=p\left(\boldsymbol{\theta} \mid \boldsymbol{Y}_{[S]}\right) / p(\boldsymbol{\theta} \mid \boldsymbol{Y})$, then $\mathrm{D}_{\phi}(S)$ is given by

$$
\mathrm{D}_{\phi}(S)=\int \phi_{\alpha}\left(R_{[S]}(\boldsymbol{\theta})\right) p(\boldsymbol{\theta} \mid \boldsymbol{Y}) d \boldsymbol{\theta},
$$

where $\phi_{\alpha}(u)$ is defined by $4\left\{1-u^{(1+\alpha) / 2}\right\} /\left(1-\alpha^{2}\right)$ for $\alpha \neq \pm 1, u \log (u)$ for $\alpha=1$, and $-\log (u)$ for $\alpha=-1$. The $\phi_{1}(\cdot)$ and $\phi_{-1}(\cdot)$ lead to the Kullback-Leibler divergence (K-L divergence), whereas $\phi(u)=\phi_{1}(u)+\phi_{-1}(u)$ leads to the symmetric K-L divergence. The $L_{1}$-distance and the $\chi^{2}$-divergence correspond to $\phi(u)=0.5|u-1|$ and $\phi(u)=(u-1)^{2}$, respectively [20].

Second, we consider Cook's posterior mode distance, denoted by $\mathrm{CP}(S)$, for quantifying the discrepancy between the posterior mode of $\boldsymbol{\theta}$ with and without the $i$ th case [10]. We define the posterior modes of $\boldsymbol{\theta}$ for the full sample $\boldsymbol{Y}$ and a subsample $\boldsymbol{Y}_{[S]}$ as $\hat{\boldsymbol{\theta}}=\operatorname{argmax}_{\boldsymbol{\theta}} \log p(\boldsymbol{\theta} \mid \boldsymbol{Y})$ and $\hat{\boldsymbol{\theta}}_{[S]}=\operatorname{argmax}_{\boldsymbol{\theta}} \log p\left(\boldsymbol{\theta} \mid \boldsymbol{Y}_{[S]}\right)$, respectively. Then, $\operatorname{CP}(S)$ is given by

$$
\mathrm{CP}(S)=\left(\hat{\boldsymbol{\theta}}_{[S]}-\hat{\boldsymbol{\theta}}\right)^{T} G_{\theta}\left(\hat{\boldsymbol{\theta}}_{[S]}-\hat{\boldsymbol{\theta}}\right),
$$

where $G_{\theta}$ is chosen to be a positive definite matrix. For instance, $G_{\theta}$ can be $J_{N}(\boldsymbol{\theta})=-\partial_{\boldsymbol{\theta}}^{2} \log p(\boldsymbol{\theta} \mid \boldsymbol{Y})=$ $-\partial_{\boldsymbol{\theta}}^{2} \log p(\boldsymbol{Y} \mid \boldsymbol{\theta})-\partial_{\boldsymbol{\theta}}^{2} \log p(\boldsymbol{\theta})$ evaluated at $\hat{\boldsymbol{\theta}}$, where $\partial_{\boldsymbol{\theta}}^{2}$ represents the second-order derivative with respect to $\boldsymbol{\theta}$. If $\partial_{\boldsymbol{\theta}}^{2} \log p(\hat{\boldsymbol{\theta}})=o_{p}\left(-\partial_{\boldsymbol{\theta}}^{2} \log p(\boldsymbol{Y} \mid \hat{\boldsymbol{\theta}})\right)$, then $\mathrm{CP}(S)$ is close to the well-known Cook's distance for deleting a set of observations [10,44]. A large value of $\mathrm{CP}(S)$ implies more influence of the set $S$ on the posterior mode.

Third, we consider Cook's posterior mean distance, denoted by $\operatorname{CM}(S)$, for quantifying the distance between the posterior mean of $\boldsymbol{\theta}$ with and without the observations in $S$. Let $\tilde{\boldsymbol{\theta}}=\int \boldsymbol{\theta} \cdot p(\boldsymbol{\theta} \mid \boldsymbol{Y}) d \boldsymbol{\theta}$ and $\tilde{\boldsymbol{\theta}}_{[S]}=\int \boldsymbol{\theta} \cdot p\left(\boldsymbol{\theta} \mid \boldsymbol{Y}_{[S]}\right) d \boldsymbol{\theta}$ be, respectively, the posterior mean of $\boldsymbol{\theta}$ for $\boldsymbol{Y}$ and $\boldsymbol{Y}_{[S]}$. The $\mathrm{CM}(S)$ is given by

$$
\operatorname{CM}(S)=\left(\tilde{\boldsymbol{\theta}}_{[S]}-\tilde{\boldsymbol{\theta}}\right)^{T} W_{\theta}\left(\tilde{\boldsymbol{\theta}}_{[S]}-\tilde{\boldsymbol{\theta}}\right),
$$

where $W_{\theta}$ is chosen to be a positive definite matrix. A large value of $\operatorname{CM}(S)$ corresponds to an influential set $S$ regarding the posterior mean.

Computationally, the proposed case influence measures can all be approximated using only MCMC samples from the full posterior distribution, $p(\boldsymbol{\theta} \mid \boldsymbol{Y})$. For diagnostic purposes, it is desirable to derive computationally feasible approximations to these case influence measures. For completeness, we include an important theoretical result regarding such approximations, whose proof can be found in [43], as follows.

Proposition 1. Assume that Assumptions $\mathrm{C}_{1-\mathrm{C}_{4} \text { in the }}$ Appendix hold and $N_{S}$ is bounded by a fixed constant. We have the following results:

(a) $D_{\phi}(S)=0.5 \ddot{\phi}(1) \times C P(S)+O_{p}\left(N^{-2}\right)=0.5 \ddot{\phi}(1) \times$ $C M(S)+O_{p}\left(N^{-2}\right)$. 
(b) $\hat{\boldsymbol{\theta}}_{[S]}=\hat{\boldsymbol{\theta}}+O_{p}\left(N^{-1}\right)=\hat{\boldsymbol{\theta}}-\left[J_{N}(\hat{\boldsymbol{\theta}})\right]^{-1} \partial_{\boldsymbol{\theta}} \log p_{S}(\hat{\boldsymbol{\theta}})[1+$ $\left.O_{p}\left(N^{-1}\right)\right]$.

(c) $\tilde{\boldsymbol{\theta}}_{[S]}=\tilde{\boldsymbol{\theta}}-\left[J_{N}(\hat{\boldsymbol{\theta}})\right]^{-1} \partial_{\boldsymbol{\theta}} \log p_{S}(\hat{\boldsymbol{\theta}})\left[1+O_{p}\left(N^{-1}\right)\right]$.

(d) $D_{\phi}(S)=0.5 \ddot{\phi}(1)\left[\partial_{\boldsymbol{\theta}} \log p_{S}(\hat{\boldsymbol{\theta}})\right]^{T}\left[J_{N}(\hat{\boldsymbol{\theta}})\right]^{-1}$ $\left[\partial_{\boldsymbol{\theta}} \log p_{S}(\hat{\boldsymbol{\theta}})\right]\left[1+O_{p}\left(N^{-1}\right)\right]$, where $\ddot{\phi}(1)=\left.\partial_{u}^{2} \phi(u)\right|_{u=1}$ and $p_{S}(\boldsymbol{\theta})=p\left(\boldsymbol{Y}_{S} \mid \boldsymbol{Y}_{[S]}, \boldsymbol{\theta}\right)$ is the conditional distribution of $\boldsymbol{Y}_{S}$ given $\boldsymbol{Y}_{[S]}$.

Proposition 1 establishes a direct connection between $\mathrm{D}_{\phi}(S), \mathrm{CP}(S)$ and $\operatorname{CM}(S)$ for any $\phi(\cdot)$ and the one-step approximation of $\hat{\boldsymbol{\theta}}_{[S]}$ and $\tilde{\boldsymbol{\theta}}_{[S]}$ within the Bayesian framework. Proposition 1 provides a theoretical and computational approximation of $\mathrm{D}_{\phi}(S)$, denoted by $\operatorname{AD}(S ; \tilde{\boldsymbol{\theta}})$, as

$$
\operatorname{AD}(S ; \tilde{\boldsymbol{\theta}})=\left[\partial_{\boldsymbol{\theta}} \log p_{S}(\tilde{\boldsymbol{\theta}})\right]^{T}\left[J_{N}(\tilde{\boldsymbol{\theta}})\right]^{-1}\left[\partial_{\boldsymbol{\theta}} \log p_{S}(\tilde{\boldsymbol{\theta}})\right] .
$$

The $\tilde{\boldsymbol{\theta}}$ and $J_{N}(\tilde{\boldsymbol{\theta}})$ can be easily computed from the MCMC samples. Moreover, it is straightforward to compute $\partial_{\boldsymbol{\theta}} \log p_{S}(\boldsymbol{\theta})=\partial_{\boldsymbol{\theta}} \log p(\boldsymbol{Y} \mid \boldsymbol{\theta})-\partial_{\boldsymbol{\theta}} \log p\left(\boldsymbol{Y}_{[S]} \mid \boldsymbol{\theta}\right)$. As an illustration, we consider a normal linear model to illustrate the calculation of Bayesian case influence measures.

Example 1. We consider a normal linear model as $\boldsymbol{Y}=\mathbf{X} \boldsymbol{\beta}+$ $\boldsymbol{\epsilon}$ or $y_{i}=\mathbf{x}_{i}^{T} \boldsymbol{\beta}+\epsilon_{i}$, where $\boldsymbol{\beta}$ and $\mathbf{x}_{i}$ are $p \times 1$ vectors, $\boldsymbol{\beta}$ is unknown, $\boldsymbol{\epsilon}=\left(\epsilon_{1}, \ldots, \epsilon_{n}\right)^{T} \sim N_{n}\left(0, \tau^{-1} \mathrm{I}\right)$, and $\tau=1 / \sigma^{2}$ is assumed known for simplicity. We consider a conjugate prior for $\boldsymbol{\beta}$ as $N_{p}\left(\boldsymbol{\mu}_{0}, \tau^{-1} \boldsymbol{\Sigma}_{0}\right)$. For a given set $S, p(\boldsymbol{\beta} \mid \boldsymbol{Y})$ and $p\left(\boldsymbol{\beta} \mid \boldsymbol{Y}_{[S]}\right)$ are, respectively, given by

$$
\boldsymbol{\beta} \mid \boldsymbol{Y} \sim N_{p}\left(\tilde{\boldsymbol{\beta}}, \tau^{-1}\left(\mathbf{X}^{T} \mathbf{X}+\boldsymbol{\Sigma}_{0}^{-1}\right)^{-1}\right)
$$

and

$$
\boldsymbol{\beta} \mid \boldsymbol{Y}_{[S]} \sim N_{p}\left(\tilde{\boldsymbol{\beta}}_{[S]}, \tau^{-1}\left(\boldsymbol{X}_{[S]}^{T} \boldsymbol{X}_{[S]}+\boldsymbol{\Sigma}_{0}^{-1}\right)^{-1}\right),
$$

where $\tilde{\boldsymbol{\beta}}=\left(\boldsymbol{X}^{T} \boldsymbol{X}+\boldsymbol{\Sigma}_{0}^{-1}\right)^{-1}\left(\boldsymbol{X}^{T} \boldsymbol{Y}+\boldsymbol{\Sigma}_{0}^{-1} \boldsymbol{\mu}_{0}\right), \tilde{\boldsymbol{\beta}}_{[S]}=$ $\left(\boldsymbol{X}_{[S]}^{T} \boldsymbol{X}_{[S]}+\boldsymbol{\Sigma}_{0}^{-1}\right)^{-1}\left(\boldsymbol{X}_{[S]}^{T} \boldsymbol{Y}_{[S]}+\boldsymbol{\Sigma}^{-1} \boldsymbol{\mu}_{0}\right), \boldsymbol{X}_{[S]}$ is $\boldsymbol{X}$ with all $\mathbf{x}_{i}$ deleted for $i \in S$, and $\boldsymbol{Y}_{[S]}$ is $\boldsymbol{Y}$ with all $y_{i}$ deleted for all $i \in S$. Note that $\boldsymbol{X}_{[S]}^{T} \boldsymbol{X}_{[S]}=\boldsymbol{X}^{T} \boldsymbol{X}-\sum_{i \in S} \mathbf{x}_{i} \mathbf{x}_{i}^{T}$ and $\boldsymbol{X}_{[S]}^{T} \boldsymbol{Y}_{[S]}=\boldsymbol{X}^{T} \boldsymbol{Y}-\sum_{i \in S} \mathbf{x}_{i} y_{i}$.

Let $S=\left\{i_{1}, \ldots, i_{N_{S}}\right\}$ and $E_{S}=\left[\mathbf{e}_{i_{1}}, \ldots, \mathbf{e}_{i_{N_{S}}}\right]$ be an $N \times N_{S}$ matrix, where $\mathbf{e}_{k}$ is an $N \times 1$ vector with a 1 at the $k$-th element and 0 elsewhere for $k \in S$. With some algebraic calculations, we have

$$
\tilde{\boldsymbol{\beta}}_{[S]}=\tilde{\boldsymbol{\beta}}-\left(\boldsymbol{X}^{T} \boldsymbol{X}+\boldsymbol{\Sigma}_{0}^{-1}\right)^{-1} \boldsymbol{X}_{S}^{T}\left(\boldsymbol{I}_{N_{S}}-P_{S}\right)^{-1} \hat{\boldsymbol{e}}_{S},
$$

where $\boldsymbol{X}_{S}=E_{S}^{T} \boldsymbol{X}, P_{S}=E_{S}^{T} P_{X 0} E_{S}$, in which $P_{X 0}=$ $\boldsymbol{X}\left(\boldsymbol{X}^{T} \boldsymbol{X}+\boldsymbol{\Sigma}_{0}^{-1}\right)^{-1} \boldsymbol{X}^{T}$, and $\hat{\boldsymbol{e}}_{S}=E_{S}^{T}(\boldsymbol{Y}-\boldsymbol{X} \tilde{\boldsymbol{\beta}})$. For the KL divergence, we get

$$
\begin{aligned}
\mathrm{D}_{\phi}(S)= & 0.5\left[\tau\left(\tilde{\boldsymbol{\beta}}-\tilde{\boldsymbol{\beta}}_{[S]}\right)^{T}\left(\boldsymbol{X}_{[S]}^{T} \boldsymbol{X}_{[S]}+\boldsymbol{\Sigma}_{0}^{-1}\right)\left(\tilde{\boldsymbol{\beta}}-\tilde{\boldsymbol{\beta}}_{[S]}\right)\right. \\
& -\log \left|\boldsymbol{I}_{p}-\left(\boldsymbol{X}^{T} \boldsymbol{X}+\boldsymbol{\Sigma}_{0}^{-1}\right)^{-1} \sum_{i \in S} \mathbf{x}_{i} \mathbf{x}_{i}^{T}\right| \\
& \left.-\operatorname{tr}\left\{\left(\boldsymbol{X}^{T} \boldsymbol{X}+\boldsymbol{\Sigma}_{0}^{-1}\right)^{-1} \sum_{i \in S} \mathbf{x}_{i} \mathbf{x}_{i}^{T}\right\}\right]
\end{aligned}
$$

Note that the posterior mode and the posterior mean are the same in this example. If we set $W_{\theta}=G_{\theta}=\tau\left(\boldsymbol{X}^{T} \boldsymbol{X}+\boldsymbol{\Sigma}_{0}^{-1}\right)$, we have

$$
\mathrm{CM}(S)=\mathrm{CP}(S)=\tau\left(\tilde{\boldsymbol{\beta}}-\tilde{\boldsymbol{\beta}}_{[S]}\right)^{T}\left(\boldsymbol{X}^{T} \boldsymbol{X}+\boldsymbol{\Sigma}_{0}^{-1}\right)\left(\tilde{\boldsymbol{\beta}}-\tilde{\boldsymbol{\beta}}_{[S]}\right) .
$$

Since $\log p_{S}(\boldsymbol{\theta})=-0.5 \tau \sum_{i \in S}\left(y_{i}-\mathbf{x}_{i}^{T} \boldsymbol{\beta}\right)^{2}$ and $J_{N}(\tilde{\boldsymbol{\theta}})=$ $\tau\left(\boldsymbol{X}^{T} \boldsymbol{X}+\boldsymbol{\Sigma}_{0}^{-1}\right)$, we have

$$
\mathrm{AD}(S ; \tilde{\boldsymbol{\theta}})=\hat{\boldsymbol{e}}_{S}^{T} \boldsymbol{X}_{S} \tau\left(\boldsymbol{X}^{T} \boldsymbol{X}+\boldsymbol{\Sigma}_{0}^{-1}\right)^{-1} \boldsymbol{X}_{S}^{T} \hat{\boldsymbol{e}}_{S} .
$$

\subsection{Cross validation and model complexity}

Bayesian case influence measures (BCIM) and crossvalidation $(\mathrm{CV})$ methods share the same strategy of splitting the data into two subsamples, but they differ from each other in validation $[33,34,13,4]$. BCIM divides the data into a target sample $\boldsymbol{Y}_{S}$ and a training sample $\boldsymbol{Y}_{[S]}$ and then estimates $\tilde{\boldsymbol{\theta}}_{[S]}$ based on the training sample $\boldsymbol{Y}_{[S]}$. Note that all development below is valid for $\hat{\boldsymbol{\theta}}_{[S]}$, but we focus on the posterior mean from here on for notational simplicity. BCIM for a given set $S$ represents the influential level of $S$. In contrast, the CV method divides the data into two subsamples including a training sample $\boldsymbol{Y}_{[S]}$ for model fitting and a validation sample $\boldsymbol{Y}_{S}$ for assessing model fit. Compared to BCIM, CV usually uses the predictive distribution $p\left(\tilde{\boldsymbol{Y}}_{S} \mid \boldsymbol{Y}_{[S]}\right)$ for model validation, where $\tilde{\boldsymbol{Y}}_{S}$ is an independent copy of $\boldsymbol{Y}_{S}$. One choice of the predictive distribution is to use $p\left(\tilde{\boldsymbol{Y}}_{S} \mid \boldsymbol{Y}_{[S]}, \tilde{\boldsymbol{\theta}}_{[S]}\right)$, where $\tilde{\boldsymbol{\theta}}_{[S]}$ is estimated based on $\boldsymbol{Y}_{[S]}$. Let $N_{B}$ be an integer and $S_{1}, \ldots, S_{N_{B}}$ is a sequence of non-empty proper subsets of $\left\{(1,1), \ldots,\left(n, m_{n}\right)\right\}$. The CV estimator of the model $p(\boldsymbol{\theta} \mid \boldsymbol{Y})$ based on $I_{S}=\left(S_{k}\right)_{1 \leq k \leq N_{B}}$ is defined by

$$
\begin{aligned}
\operatorname{CVE}\left(I_{S}\right) & =N_{B}^{-1} \sum_{S \in I_{S}} \log p\left(\boldsymbol{Y}_{S} \mid \boldsymbol{Y}_{[S]}, \tilde{\boldsymbol{\theta}}_{[S]}\right) \\
& =N_{B}^{-1} \sum_{S \in I_{S}} \log p_{S}\left(\tilde{\boldsymbol{\theta}}_{[S]}\right) .
\end{aligned}
$$

A challenging issue associated with BCIM and $\mathrm{CV}$ is to calculate the $\tilde{\boldsymbol{\theta}}_{[S]}$ 's for all possible splits. Most BCIM and $\mathrm{CV}$ methods split the data with a fixed size of the training sample. There are two major categories of splitting schemes, including exhaustive data splitting and partial data splitting. Exhaustive data splitting includes the leave- $M$-out $\mathrm{CV}$ for all $N \geq M \geq 1$. For each fixed $M$, $N_{B}=N ! /(M !(N-M) !)$ and $I_{S}$ is the set of all possible sets with a fixed size $M$. However, except for relatively small $M$, it can be computationally restrictive to calculate BCIM and $\mathrm{CV}$ for every possible subset of the $M$ data. Alternatively, one may consider partial data splitting methods, such as V-fold CV [4, 41].

An interesting question is whether there is any other connection between BCIM and CV besides the strategy of splitting the data. We can establish a connection between BCIM 
and $\mathrm{CV}$ by extending the well-known result on the asymptotic equivalence between $\mathrm{CV}$ and AIC [34]. We obtain the following theorems, whose detailed proofs can be found in the the Appendix.

THEOREM 1. Let $N_{S}$ be a fixed constant. Then we have the following results:

(i) Under Assumptions $C 1-C 4$ in the Appendix, $C V E\left(I_{S}\right)$ has an asymptotic expansion as

$$
C V E\left(I_{S}\right)=N_{B}^{-1} \sum_{S_{k} \in I_{S}} \log p_{S_{k}}(\tilde{\boldsymbol{\theta}})-M A D\left(I_{S}\right)\left[1+o_{p}(1)\right]
$$

where $M A D\left(I_{S}\right)=N_{B}^{-1} \sum_{S_{k} \in I_{S}} A D\left(S_{k} ; \tilde{\boldsymbol{\theta}}\right)$ is the mean of the $A D\left(S_{k} ; \tilde{\boldsymbol{\theta}}\right)$ 's.

(ii) Under Assumptions C1, C2, and C5 in the Appendix, we have

$$
\begin{aligned}
\operatorname{MAD}\left(I_{S}\right) & =\operatorname{tr}\left\{\left[J_{N}(\tilde{\boldsymbol{\theta}})\right]^{-1} K_{N}\left(I_{S} \mid \tilde{\boldsymbol{\theta}}\right)\right\} \\
& =N^{-1}\left\{\operatorname{tr}\left[J_{*}^{-1} K_{*}\left(I_{S}\right)\right]+o_{p}(1)\right\},
\end{aligned}
$$

where $J_{N}(\tilde{\boldsymbol{\theta}})=-\left.\partial_{\boldsymbol{\theta}}^{2} \log p(\boldsymbol{\theta} \mid \boldsymbol{Y})\right|_{\boldsymbol{\theta}=\tilde{\boldsymbol{\theta}}} \quad$ and $\quad J_{*}=$ $\lim _{N \rightarrow \infty} N^{-1} E\left[J_{N}\left(\boldsymbol{\theta}_{*}\right)\right]$, in which the expectation is taken with respect to the true data generator and $\boldsymbol{\theta}_{*}$ denotes the pseudo-true parameter [6]. Moreover, $K_{N}\left(I_{S} \mid \tilde{\boldsymbol{\theta}}\right)=\left.N_{B}^{-1} \sum_{S_{k} \in I_{S}}\left[\partial_{\boldsymbol{\theta}} \log p_{S}(\boldsymbol{\theta})\right]^{\otimes 2}\right|_{\boldsymbol{\theta}=\tilde{\boldsymbol{\theta}}}$ and

$$
K_{*}\left(I_{S}\right)=\lim _{N \rightarrow \infty}\left(N_{B}\right)^{-1} \sum_{S_{k} \in I_{S}} E\left\{\left[\partial_{\boldsymbol{\theta}} \log p_{S_{k}}\left(\boldsymbol{\theta}_{*}\right)\right]^{\otimes 2}\right\},
$$

where $\boldsymbol{a}^{\otimes 2}=\boldsymbol{a} \boldsymbol{a}^{T}$ for any vector $\boldsymbol{a}$.

Theorem 1 shows a direct connection between $\mathrm{CVE}\left(I_{S}\right)$ and $\operatorname{MAD}\left(I_{S}\right)$ and an indirect connection between $\operatorname{CVE}\left(I_{S}\right)$ and BCIM. According to Proposition 1, we can use the average of the BCIMs to approximate $\operatorname{MAD}\left(I_{S}\right)$ as follows:

$$
\operatorname{MAD}\left(I_{S}\right)=N_{B}^{-1} \sum_{S_{k} \in I_{S}} \operatorname{CP}\left(S_{k}\right)+O_{p}\left(N^{-1}\right) .
$$

A similar approximation also holds for both $\operatorname{CM}(S)$ and $D_{\phi}(S)$. Moreover, $\operatorname{MAD}\left(I_{S}\right)$ is always nonnegative. Throughout the paper, based on $\operatorname{MAD}\left(I_{S}\right)$ and their approximations, we define the Bayesian case-deletion model complexity (BCMC) measures as

$$
\operatorname{BCMC}\left(I_{S}\right)=N N_{S}^{-1} \times \operatorname{MAD}\left(I_{S}\right) \approx N_{S}^{-1} \operatorname{tr}\left[J_{*}^{-1} K_{*}\left(I_{S}\right)\right] .
$$

We will show below that our BCMC measures can be regarded as a generalization of many existing measures of model complexity. We first consider single cluster deletion (or the leave-one-out CV) for clustered data, in which the $\boldsymbol{Y}_{i}$ 's are independent for different $i$, but the components in each $\boldsymbol{Y}_{i}$ may be correlated. For the leave-one-out $\mathrm{CV}$, we denote $I_{L O O}=\{\{1\}, \ldots,\{n\}\}$. In this case, we have $N_{B}=n, p_{\{i\}}(\boldsymbol{\theta})=p\left(\boldsymbol{Y}_{i} \mid \boldsymbol{\theta}\right)$,

$$
K_{N}\left(I_{L O O} \mid \tilde{\boldsymbol{\theta}}\right)
$$

$$
\begin{aligned}
& =\left.n^{-1} \sum_{i=1}^{n}\left\{\partial_{\boldsymbol{\theta}} \log p\left(\boldsymbol{Y}_{i} \mid \boldsymbol{\theta}\right)\right\}^{\otimes 2}\right|_{\boldsymbol{\theta}=\tilde{\boldsymbol{\theta}}} \\
& \rightarrow^{p} \quad K_{*}\left(I_{L O O}\right)=\lim _{n \rightarrow \infty} E\left\{K_{N}\left(I_{L O O} \mid \boldsymbol{\theta}_{*}\right)\right\},
\end{aligned}
$$

and

$$
\begin{aligned}
& J_{N}(\tilde{\boldsymbol{\theta}}) \\
&=-\left.n^{-1}\left[\sum_{i=1}^{n} \partial_{\boldsymbol{\theta}}^{2} \log p\left(\boldsymbol{Y}_{i} \mid \boldsymbol{\theta}\right)+\partial_{\boldsymbol{\theta}}^{2} \log p(\boldsymbol{\theta})\right]\right|_{\boldsymbol{\theta}=\tilde{\boldsymbol{\theta}}} \\
& \rightarrow^{p} \quad J_{*}=\lim _{N \rightarrow \infty} E\left\{J_{N}\left(\boldsymbol{\theta}_{*}\right)\right\},
\end{aligned}
$$

where $\rightarrow^{p}$ denotes convergence in probability. Let $p_{*}=$ $\operatorname{BCMC}\left(I_{L O O}\right)$ in this case. Using a uniform improper prior for $\boldsymbol{\theta}$, that is, $\partial_{\boldsymbol{\theta}}^{2} \log p(\boldsymbol{\theta})=0, p_{*}$ is the measure of model complexity in TIC. Furthermore, if the model $p(\boldsymbol{Y} \mid \boldsymbol{\theta})$ is correctly specified, then $p_{*}$ reduces to $p$, the number of parameters, and $\operatorname{MAD}\left(I_{L O O}\right)=p+o_{p}(1)$. In this case, $p$ is the measure of model complexity in AIC. For general priors, $p_{*}$ is the effective number of parameters in the network information criterion (NIC) $[27,30]$. Moreover, $\operatorname{MAD}\left(I_{L O O}\right)$ is also associated with the effective number of parameters, denoted by $p_{D}$, in DIC, where $p_{D}=E_{\theta \mid Y}[-2 \log p(\boldsymbol{Y} \mid \boldsymbol{\theta})]+$ $2 \log [p(\boldsymbol{Y} \mid \tilde{\boldsymbol{\theta}})]$. Under the two conditions of approximately normal likelihoods and a uniform improper prior for $\boldsymbol{\theta}$, it can be shown that $p_{D}=\operatorname{tr}\left\{J_{N}(\tilde{\boldsymbol{\theta}}) E\left[(\boldsymbol{\theta}-\tilde{\boldsymbol{\theta}})^{\otimes 2}\right]\right\}+$ $o_{p}(1)$ [32]. Moreover, using the fact that $E\left[(\boldsymbol{\theta}-\tilde{\boldsymbol{\theta}})^{\otimes 2}\right]=$ $J_{N}\left(\boldsymbol{\theta}_{*}\right)^{-1} K_{N}\left(I_{L O O} \mid \boldsymbol{\theta}_{*}\right) J_{N}\left(\boldsymbol{\theta}_{*}\right)^{-1}\left[1+o_{p}(1)\right][6]$, we can obtain the following connections between $p_{D}$ and $p_{*}: p_{D}=$ $p_{*}+o_{p}(1)$. Thus, $\operatorname{MAD}\left(I_{L O O}\right)$ has many of the same properties as $p_{D}[32]$. We also note that $\operatorname{MAD}\left(I_{L O O}\right)$ is always nonnegative, whereas $p_{D}$ is not.

Second, we consider multiple cluster deletion (or the leave-M clusters-out CV) for clustered data. Specifically, we focus on deleting every possible subset of the data from $M$ clusters and using it for validation. Let $I_{L M O}$ be the set of all $N_{B}=\left(\begin{array}{c}n \\ M\end{array}\right)$ subsets with $M$ clusters. If we set $S_{1}=\left\{\left\{i_{1}\right\}, \ldots,\left\{i_{M}\right\}\right\}$, then we have

$$
\begin{aligned}
& E\left\{\left[\partial_{\boldsymbol{\theta}} \log p_{S_{1}}\left(\boldsymbol{\theta}_{*}\right)\right]^{\otimes 2}\right\} \\
= & \sum_{i_{k}, i_{k}^{\prime}} E\left\{\partial_{\boldsymbol{\theta}} \log p\left(\boldsymbol{Y}_{i_{k}} \mid \boldsymbol{\theta}_{*}\right) \partial_{\boldsymbol{\theta}} \log p\left(\boldsymbol{Y}_{i_{k}^{\prime}} \mid \boldsymbol{\theta}_{*}\right)^{T}\right\} \\
= & \sum_{k=1}^{M} E\left\{\partial_{\boldsymbol{\theta}} \log p\left(\boldsymbol{Y}_{i_{k}} \mid \boldsymbol{\theta}_{*}\right)^{\otimes 2}\right\} .
\end{aligned}
$$

Therefore, by doing exhaustive data splitting, we have

$$
\begin{aligned}
& N_{B}^{-1} \sum_{S_{k} \in I_{S}} E\left\{\left[\partial_{\boldsymbol{\theta}} \log p_{S_{k}}\left(\boldsymbol{\theta}_{*}\right)\right]^{\otimes 2}\right\} \\
= & \frac{M}{n} \sum_{i=1}^{n} E\left\{\partial_{\boldsymbol{\theta}} \log p\left(\boldsymbol{Y}_{i} \mid \boldsymbol{\theta}_{*}\right)^{\otimes 2}\right\},
\end{aligned}
$$


which yields that $\operatorname{MAD}\left(I_{L M O}\right)=M \times \operatorname{MAD}\left(I_{L O O}\right)$. If $m_{1}=$ $\cdots=m_{n}$, then $\operatorname{BCMC}\left(I_{L M O}\right)=\operatorname{BCMC}\left(I_{L O O}\right)$. Similar discussions also hold for V-fold CV [4, 41].

Third, we consider single observation deletion $I_{S O}=$ $\left\{\{(1,1)\}, \ldots,\left\{\left(n, m_{n}\right)\right\}\right\}$ and examine $\operatorname{MAD}\left(I_{S O}\right)$ for clustered data. We have $N_{B}=N=\sum_{i=1}^{n} m_{i}$ and

$$
\partial_{\boldsymbol{\theta}} \log p_{[(i, j)]}(\boldsymbol{\theta})=\partial_{\boldsymbol{\theta}} \log p\left(\boldsymbol{Y}_{i} \mid \boldsymbol{\theta}\right)-\partial_{\boldsymbol{\theta}} \log p\left(\boldsymbol{Y}_{i,[(i, j)]} \mid \boldsymbol{\theta}\right),
$$

where $\boldsymbol{Y}_{i,[(i, j)]}$ denotes $\boldsymbol{Y}_{i}$ with $y_{i, j}$ deleted. Then, $K_{N}\left(I_{S O} \mid \tilde{\boldsymbol{\theta}}\right)$ is given by

$$
\begin{aligned}
& \sum_{i=1}^{n} m_{i}\left\{\partial_{\boldsymbol{\theta}} \log p\left(\boldsymbol{Y}_{i} \mid \tilde{\boldsymbol{\theta}}\right)\right\}^{\otimes 2} \\
- & \sum_{i=1}^{n} \partial_{\boldsymbol{\theta}} \log p\left(\boldsymbol{Y}_{i} \mid \tilde{\boldsymbol{\theta}}\right)\left\{\sum_{j=1}^{m_{i}} \partial_{\boldsymbol{\theta}} \log p\left(\boldsymbol{Y}_{i,[i, j]} \mid \tilde{\boldsymbol{\theta}}\right)\right\}^{T} \\
- & \sum_{i=1}^{n}\left\{\sum_{j=1}^{m_{i}} \partial_{\boldsymbol{\theta}} \log p\left(\boldsymbol{Y}_{i,[i, j]} \mid \tilde{\boldsymbol{\theta}}\right)\right\}\left[\partial_{\boldsymbol{\theta}} \log p\left(\boldsymbol{Y}_{i} \mid \tilde{\boldsymbol{\theta}}\right)\right]^{T} \\
+ & \sum_{i=1}^{n} \sum_{j=1}^{m_{i}}\left\{\partial_{\boldsymbol{\theta}} \log p\left(\boldsymbol{Y}_{i,[i, j]} \mid \tilde{\boldsymbol{\theta}}\right)\right\}^{\otimes 2} .
\end{aligned}
$$

Moreover, $p_{*}=\operatorname{tr}\left[J_{*}^{-1} K_{*}\left(I_{S O}\right)\right]$ can be regarded as a measure of model complexity for clustered data. Even if the model $p(\boldsymbol{Y} \mid \boldsymbol{\theta})$ is correctly specified, $p_{*}$ does not reduce to $p$, the number of parameters, and $\operatorname{MAD}\left(I_{S O}\right) \neq p+o_{p}(1)$. Compared with $p$ as the measure of model complexity in AIC, $p_{*}=\operatorname{tr}\left[J_{*}^{-1} K_{*}\left(I_{S O}\right)\right]$ accounts for the correlation structure in the clustered data. Although one may consider other case deletion mechanisms, we omit them here for brevity.

Example 1 (continued). In this case, we have

$$
\begin{gathered}
\operatorname{CVE}\left(I_{S}\right)=-0.5 \tau N_{B}^{-1} \sum_{S_{k} \in I_{S}} \sum_{i \in S_{k}}\left(y_{i}-\mathbf{x}_{i}^{T} \tilde{\boldsymbol{\beta}}_{[S]}\right)^{2}, \\
N_{B}^{-1} \sum_{S_{k} \in I_{S}} \log p_{S_{k}}(\tilde{\boldsymbol{\theta}})=-0.5 \tau N_{B}^{-1} \sum_{S_{k} \in I_{S}} \sum_{i \in S_{k}}\left(y_{i}-\mathbf{x}_{i}^{T} \tilde{\boldsymbol{\beta}}\right)^{2}, \\
\operatorname{MAD}\left(I_{S}\right)=N_{B}^{-1} \sum_{S_{k} \in I_{S}} \hat{\boldsymbol{e}}_{S_{k}}^{T} \boldsymbol{X}_{S_{k}} \tau\left(\boldsymbol{X}^{T} \boldsymbol{X}+\boldsymbol{\Sigma}_{0}^{-1}\right)^{-1} \boldsymbol{X}_{S_{k}}^{T} \hat{\boldsymbol{e}}_{S_{k}} .
\end{gathered}
$$

According to Theorem 1, we have

$$
=\begin{aligned}
& \operatorname{MAD}\left(I_{S}\right) \\
& N_{B}^{-1} \operatorname{tr}\left(\left(\boldsymbol{X}^{T} \boldsymbol{X}+\boldsymbol{\Sigma}_{0}^{-1}\right)^{-1} \sum_{S_{k} \in I_{S}} \boldsymbol{X}_{S_{k}}^{T} \boldsymbol{X}_{S_{k}}\right)\left[1+o_{p}(1)\right] .
\end{aligned}
$$

For the leave-one-out $\mathrm{CV}, \mathrm{BCMC}\left(I_{L O O}\right)$ can be approximated by $\sum_{i=1}^{n} p_{i i} / n$, where the $p_{i i}$ 's are the diagonal elements of $P_{X 0}$. As $\Sigma_{0}^{-1}$ converges to zero, which corresponds to a non-informative prior, $\mathrm{BCMC}\left(I_{L O O}\right)$ converges to the number of parameters in $\boldsymbol{\beta}$.

\subsection{Bayesian case-deletion information criterion}

Based on the development of $\operatorname{BCMC}\left(I_{S}\right)$ and $\operatorname{CVE}\left(I_{S}\right)$, we develop a new model selection criterion, called the Bayesian case-deletion information criterion (BCIC), to select an 'optimal' model from a pool of candidate models $\left\{M_{l}: l=1, \ldots, L\right\}$ for the same dataset. Specifically, for model $M_{l}$ and the deletion set $I_{S}$, BCIC is defined as

$$
\begin{aligned}
\operatorname{BCIC}\left(I_{S}, M_{l}\right)= & -2 \sum_{S_{k} \in I_{S}} \log p_{S_{k}}\left(\tilde{\boldsymbol{\theta}}\left(M_{l}\right), M_{l}\right) \\
& +\left(N_{B} N_{S} / N\right) C_{n}\left(I_{S}, \tilde{\boldsymbol{\theta}}\left(M_{l}\right), M_{l}\right),
\end{aligned}
$$

where $\tilde{\boldsymbol{\theta}}\left(M_{l}\right)$ is an estimator of $\boldsymbol{\theta}$ and $p_{S_{k}}\left(\boldsymbol{\theta} ; M_{l}\right)$ denotes $p\left(\boldsymbol{Y}_{S_{k}} \mid \boldsymbol{Y}_{\left[S_{k}\right]}, \boldsymbol{\theta}\right)$ under model $M_{l}$ and $C_{n}\left(I_{S}, \tilde{\boldsymbol{\theta}}\left(M_{l}\right), M_{l}\right)$ is a penalty term, which is a function of the data, the deletion set $I_{S}$, and an estimator of $\boldsymbol{\theta}\left(M_{l}\right)$. In (9), $\sum_{S_{k} \in I_{S}} \log p_{S_{k}}\left(\tilde{\boldsymbol{\theta}}\left(M_{l}\right), M_{l}\right)$ can be regarded as the conditional deviance function evaluated at $\tilde{\boldsymbol{\theta}}\left(M_{l}\right)$. We choose an 'optimal' model, denoted by $M_{\text {opt }}$, which minimizes $\operatorname{BCIC}\left(I_{S}, M_{l}\right)$, as follows:

$$
M_{\text {opt }}\left(I_{S}\right)=\operatorname{argmin}_{M_{l}: 1 \leq l \leq L} \operatorname{BCIC}\left(I_{S}, M_{l}\right) .
$$

Different forms of the model penalty $C_{n}\left(I_{S}, \tilde{\boldsymbol{\theta}}\left(M_{l}\right), M_{l}\right)$ lead to different criteria. Two popular choices of $C_{n}\left(I_{S}, \tilde{\boldsymbol{\theta}}\left(M_{l}\right), M_{l}\right)$ are the AIC-type penalty and the BIC-type penalty. For the AIC-type penalty, $C_{n}\left(I_{S}, \tilde{\boldsymbol{\theta}}\left(M_{l}\right), M_{l}\right)=C_{0} \times \operatorname{BCMC}\left(I_{S}\right)$, where $C_{0}$ is a positive scalar. In practice, similar to AIC, DIC, and TIC $[1,35,22,32]$, it is common to set $C_{0}=2$. For the BIC-type penalty, $C_{n}\left(I_{S}, \tilde{\boldsymbol{\theta}}\left(M_{l}\right), M_{l}\right)=C_{0, n} \times \operatorname{BCMC}\left(I_{S}\right)$ with $\lim _{n \rightarrow \infty} C_{0, n}=\infty$. Similar to BIC, $C_{0, n}$ is often set as $\log (N)$ or other functions of $N$. Therefore, BCIC can be regarded as a generalization of existing model selection criteria.

Different deletion sets lead to slightly different $\operatorname{BCIC}\left(I_{S}, M_{l}\right)$ for all $l$. For instance, if we consider the single cluster deletion $I_{L O O}$ and the single observation deletion $I_{S O}$, then we obtain different BCIC measures. Thus, it is possible that $M_{\text {opt }}\left(I_{S}\right)$ may vary across $I_{S}$. However, when we consider the leave-M clusters-out deletion for clustered data, we are able to obtain an invariance property of $M_{\text {opt }}\left(I_{S}\right)$. We are led to the following theorem.

THEOREM 2. Assume that the $\boldsymbol{Y}_{i}$ 's are independent and $C_{n}\left(I_{S}, \tilde{\boldsymbol{\theta}}\left(M_{l}\right), M_{l}\right)=\tilde{C}_{0, n} \times B C M C\left(I_{S}\right)$, where $\tilde{C}_{0, n}$ is independent of $I_{S}$ and $M_{l}$, but it may depend on $n$. Then, we have the following results.

(i) For the leave- $M$ clusters-out $C V$, we have $B C I C\left(I_{L M O}, M_{l}\right)=\left(\begin{array}{c}n-1 \\ M-1\end{array}\right) B C I C\left(I_{L O O}, M_{l} 0\right)$ and $M_{\text {opt }}\left(I_{L M O}\right)=M_{\text {opt }}\left(I_{L O O}\right)$ for any $M \geq 1$.

(ii) If $B C I C\left(I_{L O O}, M_{\text {opt }}\left(I_{L O O}\right)\right)-B C I C\left(I_{L O O}, M_{l}\right) \gg$ $O_{p}\left(N_{B} N^{-3 / 2}\right)$ for all $M_{l} \neq M_{o p t}\left(I_{L O O}\right)$, Assumption $C 6$ holds, and we use $M A D\left(I_{S}\right)$ to approximate $B C M C\left(I_{S}\right)$, 
then $M_{\text {opt }}\left(I_{L M O}\right)=M_{\text {opt }}\left(I_{L O O}\right)$ with probability 1 for any $M \geq 1$.

Theorem 2 shows that $\operatorname{BCIC}\left(I_{S}, M_{l}\right)$ and $M_{o p t}\left(I_{S}\right)$ are invariant for clustered data under different exhaustive splitting schemes. Due to Theorem 2, the two partitions of primary interest are now single cluster deletion $\left(I_{L O O}\right)$ and single observation deletion $\left(I_{S O}\right)$. Under $I_{L O O}$, BCIC can be simplified as

$$
\begin{aligned}
\operatorname{BCIC}\left(I_{L O O}, M_{l}\right)= & -2(n-1) \log p\left(\mathbf{Y} \mid \tilde{\boldsymbol{\theta}}\left(M_{l}\right), M_{l}\right) \\
& +n C_{0, n} M C\left(I_{L O O}\right),
\end{aligned}
$$

and under $I_{S O}$, BCIC can be simplified as

$$
\begin{aligned}
\operatorname{BCIC}\left(I_{S O}, M_{l}\right)= & -2\left[N \log p\left(\mathbf{Y} \mid \tilde{\boldsymbol{\theta}}\left(M_{l}\right), M_{l}\right)\right. \\
& \left.-\sum_{i=1}^{n} \sum_{j=1}^{m_{n}} \log p\left(Y_{i, j} \mid \tilde{\boldsymbol{\theta}}\left(M_{l}\right), M_{l}\right)\right] \\
& +N C_{0, n} M C\left(I_{S O}\right),
\end{aligned}
$$

where $M C\left(I_{L O O}\right)$ and $M C\left(I_{S O}\right)$ are shown in Section 2.2. Note that, unlike cross validation, there is not much additional computational cost associated with the BCIC procedure except the programming efforts to calculate $M C\left(I_{S}\right)$.

\section{SIMULATIONS AND REAL DATA ANALYSIS}

\subsection{Simulation studies}

In this section, several simulation studies were carried out to investigate the finite sample performance of BCIC and to compare BCIC with three existing Bayesian model selection criteria, including $\mathrm{AIC}, \mathrm{BIC}$, and DIC in linear mixed models. Specifically, we set $A I C=-2 \log p\left(Y \mid \tilde{\theta}\left(M_{l}\right)\right)+2 p$, $B I C=-2 \log p\left(Y \mid \tilde{\theta}\left(M_{l}\right)\right)+\log (N) \times p$, and $D I C=$ $-2 \log p\left(Y \mid \tilde{\theta}\left(M_{l}\right)\right)+2 p_{D}$, where $p$ is the number of parameters in the model and $p_{D}$ is the effective number of parameters, estimated by the posterior mean of the deviance minus the deviance of the posterior means. We consider both the leave-one cluster-out $\mathrm{CV}$ and the leave-one observation-out $\mathrm{CV}$, the AIC- and BIC- type penalties, and calculate their associated BCICs.

Simulated datasets were generated from a linear mixed model with a random intercept. Specifically, we consider the following true model, given by $y_{i j}=\beta_{0}+\beta_{1} x_{i j 1}+\beta_{2} x_{i j 2}+$ $b_{i}+\epsilon_{i j}$ for $i=1, \ldots, n$ and $j=1, \ldots, m_{i}$, where $x_{i j 1} \sim$ $\operatorname{Exp}(1), x_{i j 2}=j, b_{i} \sim N\left(0, \tau^{-1} \xi^{-1}\right)$, and $\epsilon_{i j} \sim N\left(0, \tau^{-1}\right)$. An additional covariate $x_{i j 3}$ was simulated from a $N(1,1)$ distribution. The true parameter values were taken to be $\beta_{0}=2, \beta_{1}=\beta_{2}=1, \tau=0.1$, and $\xi=1$ or $\xi=0.04$, for $n=10$ or $n=20$. The values of $\xi=1$ or 0.04 represent a medium or high intracluster correlation coefficient (ICC). We chose the priors as follows: $\pi\left(\beta, \tau, D^{-1}\right) \propto|D|^{-1 / 2} \tau^{-1}$ and $b \mid \tau, D \sim N_{n q}\left(0, \tau^{-1}\left(I_{n} \otimes D\right)\right)$, where $D^{-1}=\xi$ in this simulation.

We considered five candidate models as follows:

$$
\begin{aligned}
& \text { M1 } \\
& \quad \text { true model }): y_{i j} \mid x_{i j 1}, x_{i j 2} \sim N\left(\beta_{0}+\beta_{1} x_{i j 1}\right. \\
& \left.\quad+\beta_{2} x_{i j 2}+b_{i}, \tau^{-1}\right), b_{i} \sim N\left(0, \tau^{-1} \xi^{-1}\right) \\
& \text { M2 : } y_{i j} \mid x_{i j 1}, x_{i j 2} \sim N\left(\beta_{0}+\beta_{1} x_{i j 2}+b_{i}, \tau^{-1}\right) \\
& \quad b_{i} \sim N\left(0, \tau^{-1} \xi^{-1}\right) \\
& \text { M3 : } y_{i j} \mid x_{i j 1}, x_{i j 2}, x_{i j 3} \sim N\left(\beta_{0}+\beta_{1} x_{i j 1}\right. \\
& \left.\quad+\beta_{2} x_{i j 2}+\beta_{3} x_{i j 3}+b_{i}, \tau^{-1}\right), b_{i} \sim N\left(0, \tau^{-1} \xi^{-1}\right) \\
& \text { M4 : } y_{i j} \mid x_{i j 1}, x_{i j 2}, x_{i j 3} \sim N\left(\beta_{0}+\beta_{1} x_{i j 1}\right. \\
& \left.\quad+\beta_{2} x_{i j 2}+\beta_{3} x_{i j 2} x_{i j 3}+b_{i}, \tau^{-1}\right) \\
& \quad b_{i} \sim N\left(0, \tau^{-1} \xi^{-1}\right) ; \\
& \text { M5 : } y_{i j} \mid x_{i j 1}, x_{i j 2}, x_{i j 3} \sim N\left(\beta_{0}+\beta_{1} x_{i j 1}\right. \\
& \left.\quad+\beta_{2} x_{i j 2}+\beta_{3} x_{i j 3}+\beta_{4} x_{i j 2} x_{i j 3}+b_{i}, \tau^{-1}\right) \\
& \quad b_{i} \sim N\left(0, \tau^{-1} \xi^{-1}\right) .
\end{aligned}
$$

We generated 1,000 simulated datasets from M1 and then calculated AIC, BIC, DIC, and BCIC for the five candidate models M1-M5.

Tables 1 and 2 show the number of times out of 1,000 simulations that each rank was achieved for the true model M1 for all model selection criteria. The columns correspond to the rankings of $\mathrm{AIC}, \mathrm{BIC}$, and DIC under different settings, and the rows corresponds to the proposed BCIC criteria for different choices of $k$ and $I_{S}$. Table 1 provides the results for the setting with $n=10$ and $m_{i}$ varying between 3 and 10 , representing deletion of moderate numbers of observations in an unbalanced design, whereas Table 2 shows the results for the setting with $n=20$ and $m_{i}$ varying between 3 and 15 , a setup with deletion of a relatively large number of observations in an unbalanced design. In the simulation, 1,000 burn-in and 5,000 Gibbs samples were used in the calculation. The convergence of the Gibbs sampler was checked by trace plots, but was not included here.

With $n=10, m_{i}$ from $[3,10]$, and $\mathrm{ICC}=0.5, \mathrm{M} 1$ was ranked number one $556(=349+118+53+33+3)$ times by AIC, 548 times by BIC, 467 times by DIC, 390 times by $\mathrm{BCIC}\left(I_{L O O}\right)$ and 544 times by $\mathrm{BCIC}\left(I_{S O}\right)$ for $C_{0}=2$, and 462 times by $\operatorname{BCIC}\left(I_{L O O}\right)$ and 561 times by $\operatorname{BCIC}\left(I_{S O}\right)$ for $C_{0, n}=\log (N)$, respectively. With ICC increasing to 0.96 , M1 was ranked number one 675 times by AIC, 887 times by BIC, 536 times by DIC, 452 times by $\operatorname{BCIC}\left(I_{L O O}\right)$ and 652 times by $\operatorname{BCIC}\left(I_{S O}\right)$ for $C_{0}=2$, and 582 times by BCIC $\left(I_{L O O}\right)$ and 875 times by $\operatorname{BCIC}\left(I_{S O}\right)$ for $C_{0, n}=\log (N)$, respectively.

With $n=20, m_{i}$ from $[3,15]$, and $\mathrm{ICC}=0.5$, M1 was ranked number one 719 times by AIC, 847 times by BIC, 571 times by DIC, 614 times by $\operatorname{BCIC}\left(I_{L O O}\right)$ and 724 times by $\operatorname{BCIC}\left(I_{S O}\right)$ for $C_{0}=2$, and 737 times by BCIC $\left(I_{L O O}\right)$ and 837 times by $\operatorname{BCIC}\left(I_{S O}\right)$ for $C_{0, n}=\log (N)$, respectively. With ICC increasing to 0.96, M1 was ranked number one 727 times by AIC, 966 times by BIC, 587 times by DIC, 
Table 1. Ranks of the true model M1 for BCIC, AIC, BIC, and DIC in the linear mixed model. The number of clusters is $n=10$ and the number of individuals within each cluster, $m_{i}$, varies between 3 and 10. Two levels of the intracluster correlation coefficient (ICC) are considered

\begin{tabular}{|c|c|c|c|c|c|c|c|c|c|c|c|c|c|c|c|}
\hline \multirow{2}{*}{$\begin{array}{l}\text { BCIC } \\
\text { Rank }\end{array}$} & \multicolumn{5}{|c|}{ AIC } & \multicolumn{5}{|c|}{$\mathrm{BIC}$} & \multicolumn{5}{|c|}{ DIC } \\
\hline & 1 & 2 & 3 & 4 & 5 & 1 & 2 & 3 & 4 & 5 & 1 & 2 & 3 & 4 & 5 \\
\hline & \multicolumn{15}{|c|}{$\xi=1(\mathrm{ICC}=0.5)$} \\
\hline & \multicolumn{15}{|c|}{$\operatorname{BCIC}\left(I_{L O O}\right)$ with $C_{0}=2$} \\
\hline 1 & 349 & 33 & 7 & 1 & 0 & 285 & 104 & 0 & 1 & 0 & 249 & 78 & 45 & 17 & 1 \\
\hline 2 & 118 & 96 & 15 & 5 & 0 & 121 & 111 & 2 & 0 & 0 & 109 & 82 & 22 & 19 & 2 \\
\hline 3 & 53 & 48 & 55 & 17 & 0 & 84 & 72 & 14 & 3 & 0 & 62 & 48 & 45 & 16 & 2 \\
\hline 4 & 33 & 40 & 38 & 36 & 4 & 57 & 69 & 14 & 8 & 3 & 35 & 55 & 36 & 20 & 5 \\
\hline \multirow[t]{2}{*}{5} & 3 & 16 & 12 & 11 & 9 & 1 & 38 & 7 & 3 & 2 & 12 & 10 & 15 & 9 & 5 \\
\hline & \multicolumn{15}{|c|}{$\operatorname{BCIC}\left(I_{S O}\right)$ with $C_{0}=2$} \\
\hline 1 & 504 & 33 & 5 & 2 & 0 & 393 & 151 & 0 & 0 & 0 & 361 & 112 & 47 & 23 & 1 \\
\hline 2 & 43 & 164 & 27 & 1 & 0 & 95 & 140 & 0 & 0 & 0 & 69 & 98 & 43 & 22 & 3 \\
\hline 3 & 7 & 28 & 78 & 16 & 0 & 45 & 66 & 16 & 2 & 0 & 30 & 46 & 39 & 12 & 2 \\
\hline 4 & 2 & 8 & 14 & 50 & 2 & 15 & 32 & 17 & 9 & 3 & 6 & 14 & 32 & 20 & 4 \\
\hline \multirow[t]{2}{*}{5} & 0 & 0 & 3 & 1 & 11 & 0 & 5 & 4 & 4 & 2 & 1 & 3 & 2 & 4 & 5 \\
\hline & \multicolumn{15}{|c|}{$\operatorname{BCIC}\left(I_{L O O}\right)$ with $C_{0, n}=\log (N)$} \\
\hline 1 & 360 & 64 & 28 & 10 & 0 & 360 & 98 & 2 & 2 & 0 & 265 & 113 & 58 & 23 & 3 \\
\hline 2 & 125 & 95 & 40 & 14 & 0 & 98 & 165 & 9 & 0 & 2 & 117 & 87 & 43 & 23 & 4 \\
\hline 3 & 47 & 41 & 30 & 17 & 5 & 60 & 65 & 8 & 5 & 2 & 48 & 40 & 32 & 17 & 3 \\
\hline 4 & 20 & 18 & 17 & 19 & 3 & 21 & 40 & 11 & 4 & 1 & 20 & 22 & 20 & 12 & 3 \\
\hline \multirow[t]{2}{*}{5} & 4 & 15 & 12 & 10 & 5 & 9 & 26 & 7 & 4 & 0 & 17 & 11 & 10 & 6 & 2 \\
\hline & \multicolumn{15}{|c|}{$\operatorname{BCIC}\left(I_{S O}\right)$ with $C_{0, n}=\log (N)$} \\
\hline 1 & 420 & 86 & 45 & 10 & 0 & 497 & 59 & 4 & 1 & 0 & 334 & 133 & 69 & 23 & 2 \\
\hline 2 & 136 & 140 & 66 & 23 & 1 & 45 & 314 & 7 & 0 & 0 & 131 & 129 & 62 & 40 & 4 \\
\hline 3 & 0 & 7 & 15 & 19 & 5 & 4 & 17 & 20 & 4 & 1 & 2 & 9 & 24 & 6 & 5 \\
\hline 4 & 0 & 0 & 1 & 17 & 3 & 2 & 4 & 6 & 7 & 2 & 0 & 1 & 7 & 11 & 2 \\
\hline \multirow[t]{3}{*}{5} & 0 & 0 & 0 & 1 & 4 & 0 & 0 & 0 & 3 & 2 & 0 & 1 & 1 & 1 & 2 \\
\hline & \multicolumn{15}{|c|}{$\xi=0.04(\mathrm{ICC}=0.96)$} \\
\hline & \multicolumn{15}{|c|}{$\operatorname{BCIC}\left(I_{L O O}\right)$ with $C_{0}=2$} \\
\hline 1 & 431 & 16 & 5 & 0 & 0 & 451 & 1 & 0 & 0 & 0 & 306 & 98 & 45 & 3 & 0 \\
\hline 2 & 137 & 50 & 11 & 2 & 0 & 191 & 9 & 0 & 0 & 0 & 119 & 48 & 29 & 4 & 0 \\
\hline 3 & 69 & 37 & 41 & 11 & 0 & 132 & 21 & 3 & 2 & 0 & 60 & 44 & 42 & 12 & 0 \\
\hline 4 & 38 & 28 & 41 & 76 & 0 & 113 & 34 & 28 & 8 & 0 & 51 & 41 & 56 & 35 & 0 \\
\hline \multirow[t]{2}{*}{5} & 0 & 0 & 1 & 5 & 0 & 0 & 0 & 4 & 2 & 0 & 0 & 1 & 2 & 3 & 0 \\
\hline & $\mathrm{BCI}$ & SO) & $C_{0}$ & & & & & & & & & & & & \\
\hline 1 & 619 & 26 & 7 & 0 & 0 & 650 & 2 & 0 & 0 & 0 & 449 & 144 & 52 & 7 & 0 \\
\hline 2 & 51 & 78 & 17 & 4 & 0 & 139 & 11 & 0 & 0 & 0 & 61 & 48 & 34 & 7 & 0 \\
\hline 3 & 5 & 24 & 58 & 10 & 0 & 73 & 19 & 5 & 0 & 0 & 18 & 27 & 41 & 11 & 0 \\
\hline 4 & 0 & 3 & 17 & 80 & 0 & 25 & 33 & 30 & 12 & 0 & 8 & 13 & 47 & 32 & 0 \\
\hline & $\mathrm{BCI}$ & LOO) & $\mathrm{h} C$ & $=\log$ & & & & & & & & & & & \\
\hline 1 & 502 & 53 & 19 & 8 & 0 & 572 & 7 & 2 & 1 & 0 & 359 & 135 & 78 & 10 & 0 \\
\hline 2 & 106 & 44 & 26 & 13 & 0 & 163 & 18 & 7 & 1 & 0 & 100 & 43 & 36 & 10 & 0 \\
\hline 3 & 39 & 18 & 35 & 23 & 0 & 84 & 23 & 7 & 1 & 0 & 45 & 22 & 34 & 14 & 0 \\
\hline 4 & 28 & 16 & 18 & 41 & 0 & 68 & 14 & 15 & 6 & 0 & 31 & 31 & 23 & 18 & 0 \\
\hline 5 & 0 & 0 & 1 & 9 & 0 & 0 & 3 & 4 & 3 & 0 & 1 & 1 & 3 & 5 & 0 \\
\hline & $\mathrm{BCI}$ & SO) $\mathrm{x}$ & $C_{0,}$ & $\log ($ & & & & & & & & & & & \\
\hline 1 & 670 & 117 & 69 & 19 & 0 & 852 & 18 & 5 & 0 & 0 & 520 & 215 & 115 & 25 & 0 \\
\hline 2 & 5 & 14 & 23 & 26 & 0 & 29 & 35 & 4 & 0 & 0 & 13 & 13 & 27 & 15 & 0 \\
\hline 3 & 0 & 0 & 7 & 32 & 0 & 5 & 11 & 18 & 5 & 0 & 3 & 3 & 21 & 12 & 0 \\
\hline 4 & 0 & 0 & 0 & 17 & 0 & 1 & 1 & 8 & 7 & 0 & 0 & 1 & 11 & 5 & 0 \\
\hline
\end{tabular}


Table 2. Ranks of the true model M1 for BCIC, AIC, BIC, and DIC in the linear mixed model. The number of clusters is $n=20$ and the number of individuals within each cluster, $m_{i}$, varies between 3 and 15 . Two levels of the intracluster correlation coefficient (ICC) are considered

\begin{tabular}{|c|c|c|c|c|c|c|c|c|c|c|c|c|c|c|c|}
\hline \multirow{2}{*}{$\begin{array}{l}\text { BCIC } \\
\text { Rank }\end{array}$} & \multicolumn{5}{|c|}{ AIC } & \multicolumn{5}{|c|}{$\mathrm{BIC}$} & \multicolumn{5}{|c|}{ DIC } \\
\hline & 1 & 2 & 3 & 4 & 5 & 1 & 2 & 3 & 4 & 5 & 1 & 2 & 3 & 4 & 5 \\
\hline & \multicolumn{15}{|c|}{$\xi=1(\mathrm{ICC}=0.5)$} \\
\hline & \multicolumn{15}{|c|}{$\operatorname{BCIC}\left(I_{L O O}\right)$ with $C_{0}=2$} \\
\hline 1 & 581 & 23 & 9 & 1 & 0 & 557 & 57 & 0 & 0 & 0 & 427 & 113 & 68 & 6 & 0 \\
\hline 2 & 95 & 44 & 15 & 2 & 0 & 126 & 30 & 0 & 0 & 0 & 84 & 42 & 25 & 5 & 0 \\
\hline 3 & 29 & 18 & 42 & 14 & 0 & 88 & 14 & 1 & 0 & 0 & 35 & 38 & 27 & 2 & 1 \\
\hline 4 & 14 & 17 & 35 & 59 & 0 & 76 & 26 & 21 & 2 & 0 & 25 & 24 & 56 & 19 & 1 \\
\hline \multirow[t]{2}{*}{5} & 0 & 0 & 0 & 0 & 1 & 0 & 1 & 0 & 0 & 0 & 0 & 0 & 0 & 1 & 0 \\
\hline & \multicolumn{15}{|c|}{$\operatorname{BCIC}\left(I_{S O}\right)$ with $C_{0}=2$} \\
\hline 1 & 690 & 28 & 6 & 0 & 0 & 662 & 62 & 0 & 0 & 0 & 503 & 139 & 76 & 6 & 0 \\
\hline 2 & 24 & 61 & 21 & 4 & 0 & 82 & 28 & 0 & 0 & 0 & 41 & 41 & 23 & 5 & 0 \\
\hline 3 & 5 & 12 & 66 & 10 & 0 & 79 & 13 & 1 & 0 & 0 & 24 & 30 & 37 & 2 & 0 \\
\hline \multirow[t]{2}{*}{4} & 0 & 1 & 8 & 62 & 1 & 24 & 25 & 21 & 2 & 0 & 3 & 7 & 40 & 20 & 2 \\
\hline & \multicolumn{15}{|c|}{$\operatorname{BCIC}\left(I_{L O O}\right)$ with $C_{0, n}=\log (N)$} \\
\hline 1 & 598 & 59 & 56 & 24 & 0 & 705 & 28 & 4 & 0 & 0 & 452 & 159 & 112 & 14 & 0 \\
\hline 2 & 102 & 34 & 29 & 15 & 1 & 101 & 79 & 1 & 0 & 0 & 93 & 44 & 34 & 9 & 1 \\
\hline 3 & 15 & 6 & 12 & 22 & 0 & 33 & 10 & 11 & 1 & 0 & 20 & 10 & 18 & 6 & 1 \\
\hline 4 & 4 & 3 & 4 & 13 & 0 & 8 & 11 & 5 & 0 & 0 & 6 & 4 & 10 & 4 & 0 \\
\hline \multirow[t]{2}{*}{5} & 0 & 0 & 0 & 2 & 0 & 0 & 0 & 1 & 1 & 0 & 0 & 0 & 2 & 0 & 0 \\
\hline & \multicolumn{15}{|c|}{$\operatorname{BCIC}\left(I_{S O}\right)$ with $C_{0, n}=\log (N)$} \\
\hline 1 & 639 & 78 & 86 & 34 & 0 & 817 & 18 & 2 & 0 & 0 & 508 & 182 & 132 & 15 & 0 \\
\hline 2 & 80 & 24 & 13 & 22 & 1 & 29 & 104 & 7 & 0 & 0 & 62 & 35 & 28 & 13 & 2 \\
\hline 3 & 0 & 0 & 2 & 16 & 0 & 1 & 6 & 11 & 0 & 0 & 1 & 0 & 12 & 5 & 0 \\
\hline \multirow[t]{3}{*}{4} & 0 & 0 & 0 & 4 & 0 & 0 & 0 & 2 & 2 & 0 & 0 & 0 & 4 & 0 & 0 \\
\hline & \multicolumn{15}{|c|}{$\xi=0.04(\mathrm{ICC}=0.96)$} \\
\hline & \multicolumn{15}{|c|}{$\operatorname{BCIC}\left(I_{L O O}\right)$ with $C_{0}=2$} \\
\hline 1 & 584 & 16 & 10 & 0 & 0 & 610 & 0 & 0 & 0 & 0 & 443 & 107 & 57 & 3 & 0 \\
\hline 2 & 85 & 39 & 20 & 2 & 0 & 145 & 1 & 0 & 0 & 0 & 78 & 39 & 26 & 3 & 0 \\
\hline 3 & 47 & 31 & 57 & 9 & 0 & 140 & 2 & 2 & 0 & 0 & 52 & 43 & 42 & 7 & 0 \\
\hline \multirow[t]{2}{*}{4} & 11 & 12 & 31 & 45 & 0 & 71 & 19 & 8 & 1 & 0 & 14 & 31 & 45 & 9 & 0 \\
\hline & \multicolumn{15}{|c|}{$\operatorname{BCIC}\left(I_{S O}\right)$ with $C_{0}=2$} \\
\hline 1 & 714 & 27 & 8 & 0 & 0 & 749 & 0 & $\overline{0}$ & 0 & 0 & 537 & 136 & 73 & 3 & 0 \\
\hline 2 & 12 & 59 & 19 & 0 & 0 & 89 & 1 & 0 & 0 & 0 & 28 & 35 & 26 & 1 & 0 \\
\hline 3 & 1 & 11 & 85 & 10 & 0 & 106 & 0 & 1 & 0 & 0 & 19 & 38 & 40 & 10 & 0 \\
\hline \multirow[t]{2}{*}{4} & 0 & 1 & 6 & 46 & 0 & 22 & 21 & 9 & 1 & 0 & 3 & 11 & 31 & 8 & 0 \\
\hline & $\mathrm{BCI}$ & 200 & $\mathrm{~h} C_{\mathrm{c}}$ & $\log$ & & & & & & & & & & & \\
\hline 1 & 676 & 72 & 75 & 16 & 0 & 833 & 4 & 2 & 0 & 0 & 532 & 169 & 127 & 11 & 0 \\
\hline 2 & 39 & 20 & 23 & 16 & 0 & 89 & 7 & 1 & 1 & 0 & 40 & 31 & 22 & 5 & 0 \\
\hline 3 & 10 & 3 & 18 & 13 & 0 & 35 & 6 & 3 & 0 & 0 & 11 & 15 & 14 & 4 & 0 \\
\hline 4 & 2 & 3 & 2 & 11 & 0 & 9 & 5 & 4 & 0 & 0 & 4 & 5 & 7 & 2 & 0 \\
\hline & $\mathrm{BCI}$ & SO) & $C_{0, r}$ & $\operatorname{og}(1$ & & & & & & & & & & & \\
\hline 1 & 727 & 98 & 112 & 33 & 0 & 960 & 9 & 1 & 0 & 0 & 586 & 213 & 155 & 16 & 0 \\
\hline 2 & 0 & 0 & 6 & 14 & 0 & 5 & 11 & 4 & 0 & 0 & 1 & 7 & 9 & 3 & 0 \\
\hline 3 & 0 & 0 & 0 & 6 & 0 & 1 & 2 & 3 & 0 & 0 & 0 & 0 & 4 & 2 & 0 \\
\hline 4 & 0 & 0 & 0 & 3 & 0 & 0 & 0 & 2 & 1 & 0 & 0 & 0 & 2 & 1 & 0 \\
\hline
\end{tabular}

610 times by $\operatorname{BCIC}\left(I_{L O O}\right)$ and 749 times by $\operatorname{BCIC}\left(I_{S O}\right)$ for $C_{0}=2,839$ times by $\mathrm{BCIC}\left(I_{L O O}\right)$ and 970 times by $\operatorname{BCIC}\left(I_{S O}\right)$ for $C_{0, n}=\log (N)$, respectively.

These results indicate that there is no single model selection criterion that can dominate the rest. Considering differ- ent BCIC approaches, $\mathrm{BCIC}\left(I_{S O}\right)$ outperforms BCIC $\left(I_{L O O}\right)$ for both the AIC- and BIC-type penalty terms, the BIC-type penalty term outperforms the AIC-type penalty term, and $B C I C\left(I_{S O}\right)$ with the BIC-type penalty has the best performance within the BCIC model selection criteria. Compared 
with other existing model selection criteria, $\operatorname{BCIC}\left(I_{S O}\right)$ with $C_{0, n}=\log (N)$ performs similar to BIC, while $\operatorname{BCIC}\left(I_{S O}\right)$ with $C_{0}=2$ performs similar to AIC. The performance of DIC and $\operatorname{BCIC}\left(I_{L O O}\right)$ with $C_{0, n}=2$ or $C_{0, n}=\log (N)$ are among the worst in all scenarios.

\subsection{Yale infant growth data}

We consider the Yale infant growth data, which studies whether cocaine exposure during pregnancy may lead to the maltreatment of infants after birth, such as physical and sexual abuse. There are a total of 298 children with 3,176 records recruited from two exposure groups, the cocaine exposure group and the unexposed group. In this dataset, a unique feature is that different children had different numbers of visits, ranging from 2 to 30 (interquantile range: $7-13)$, as well as different patterns of visits during the study period. See Merikangas et al. [26] for a detailed description of the study design and data collection. We apply the proposed BCIC method and compare it to existing model selection criteria for these data to illustrate the application of BCIC.

Multivariate adaptive splines for the analysis of longitudinal data (MASAL) was used to analyze the Yale infant growth data in Zhang [40]. [40] selected the MASAL model

$$
y_{i j}=\boldsymbol{x}_{i j}^{T} \boldsymbol{\beta}+\epsilon_{i j},
$$

where the $\boldsymbol{x}_{i j}$ are the potential fixed effects covariates, given by

$$
\begin{aligned}
\text { (10) } \boldsymbol{x}_{i j}= & \left(1, d,(d-120)^{+},(d-200)^{+},\left(g_{a}-28\right)^{+},\right. \\
& d\left(g_{a}-28\right)^{+},(d-60)^{+}\left(g_{a}-28\right)^{+}, \\
& \left.(d-490)^{+}\left(g_{a}-28\right)^{+}, s d, s(d-120)^{+}\right)^{T},
\end{aligned}
$$

in which $d$ and $g_{a}$ are the age at visit and gestation age, respectively, and $s$ is the indicator for gender with 1 indicating a girl and 0 indicating a boy. In addition, we assume that $\boldsymbol{\epsilon}_{i}=\left(\epsilon_{i 1}, \ldots, \epsilon_{i m_{i}}\right)^{T} \sim N\left(\mathbf{0}, \Sigma_{i}(\tau, \xi)\right)$ and $\Sigma_{i}(\tau, \xi)$ is determined by the dispersion parameter $\tau$ and additional parameters $\xi$. During this reanalysis, we considered two covariance structures for $\Sigma_{i}(\tau, \xi)$, these being the $\operatorname{AR}(1)$ and compound symmetry (CS) structures, along with four sets of fixed effect covariates: (a) $\boldsymbol{x}_{i j}$; (b) $\left(\boldsymbol{x}_{i j}^{T},(d-120)^{+}\left(g_{a}-28\right)^{+}\right)$; (c) $\left(\boldsymbol{x}_{i j}^{T},(d-200)^{+}\left(g_{a}-28\right)^{+}\right)$; (d) $\left(\boldsymbol{x}_{i j}^{T}, s(d-200)^{+}\right)$. The combinations of different covariance structures and fixed effects lead to a total of eight candidate models. The same priors of Section 3.1 were used in the real data analysis. The additional correlation coefficient parameters in the AR(1) and CS structures had independent Unif $(-1,1)$ priors.

Table 3 shows the values of AIC, BIC, DIC, and four BCIC measures normalized by $N_{B}$ as well as the ranks of all eight candidate models for each criterion. The best model selected by the different criteria are slightly different - AIC, BIC, and DIC ranked the mixed model with the fixed effects of $\boldsymbol{x}_{i j}^{T} \boldsymbol{\beta}$ and the $\mathrm{AR}(1)$ covariance structure as the

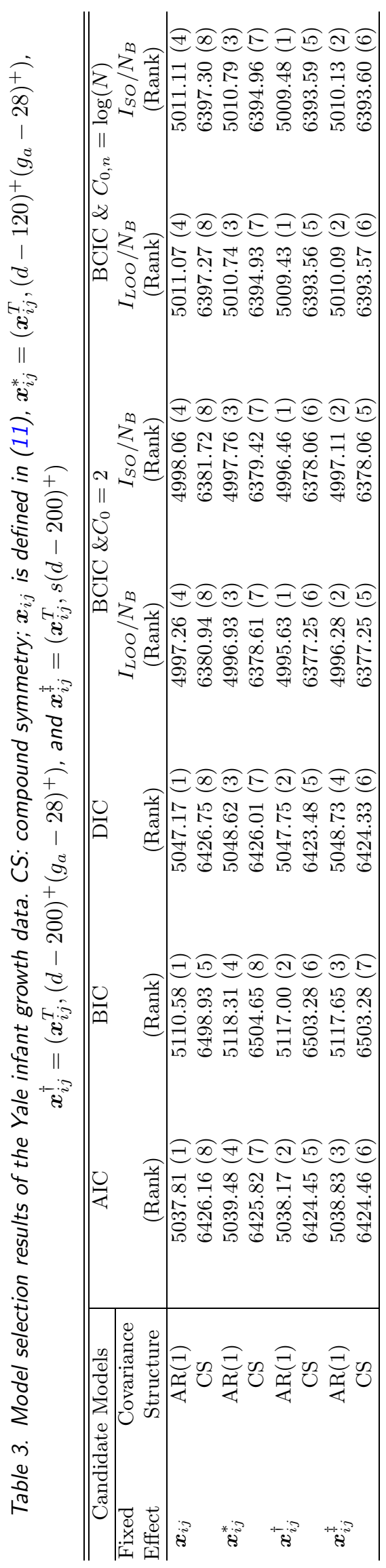


best model, and the four BCIC criteria ranked the model with the fixed effects $\left(\boldsymbol{x}_{i j}^{T},(d-200)^{+}\left(g_{a}-28\right)^{+}\right)^{T} \boldsymbol{\beta}$ and the $\mathrm{AR}(1)$ covariance structure as the best model. However, the numerical values of the criteria for the models ranked from 1-4 (all the models with the $\mathrm{AR}(1)$ covariance structure) and for the models ranked from 5-8 (all the models with the CS covariance structure) are almost indistinguishable, implying great uncertainty of the ranking decision. Furthermore, the finding that models with the $\mathrm{AR}(1)$ covariance structure always provide a better fit to these data than the models with the CS covariance structure is consistent with the longitudinal nature of this dataset.

\section{DISCUSSION}

We have systematically examined the connection between Bayesian case influence measures and Bayesian predictive methods. Based on these connections, we have developed a BCMC measure for quantifying the effective number of parameters in a given statistical model and a BCIC measure for comparing models. We have systematically investigated some properties of BCIC and BCMC and their connections with cross-validation and other existing information criteria. We have shown that BCIC is a valuable tool for Bayesian model assessment.

\section{APPENDIX: ASSUMPTIONS AND PROOFS}

We need to introduce some notation. Let $F_{N}(\boldsymbol{\theta})=$ $\partial_{\boldsymbol{\theta}} \log p(\boldsymbol{\theta} \mid \boldsymbol{Y})$ and $F_{N,[S]}(\boldsymbol{\theta})=\partial_{\boldsymbol{\theta}} \log p\left(\boldsymbol{\theta} \mid \boldsymbol{Y}_{[S]}\right)$. Under certain conditions [6], the posterior mode $\hat{\boldsymbol{\theta}}$ converges to the $\boldsymbol{\theta}_{n *}$ that minimizes $E\{-\log p(\boldsymbol{\theta} \mid \boldsymbol{Y})\}$, where the expectation is taken with respect to the true distribution of $\boldsymbol{Y}$. For simplicity, we further assume that $\boldsymbol{\theta}_{n *}=\boldsymbol{\theta}_{*}$ for all $n$. We use $\|\cdot\|$ to denote the Euclidean norm of a vector or a matrix and use $\lambda_{\max }(A)$ and $\lambda_{\min }(A)$ to denote the largest and smallest eigenvalues of a symmetric matrix $A$, respectively. We use the mathematical symbols (e.g., $O\left(N^{-1}\right)$ ) and the stochastic-order symbols, such as $O_{p}(1), o_{p}(1)$, and $O_{p}\left(N^{-1}\right)$ throughout.

The following assumptions are needed to facilitate the technical details, although they are not the weakest possible conditions. Since we develop all results for general parametric models, we only assume several high-level assumptions as follows.

Assumption $C 1$. $\hat{\boldsymbol{\theta}}$ and $\hat{\boldsymbol{\theta}}_{[S]}$ for all $S$ are consistent estimates of $\boldsymbol{\theta}_{*} \in \Theta^{o}$.

Assumption C2. Let $\Delta(\boldsymbol{\theta})=\boldsymbol{\theta}-\boldsymbol{\theta}_{*}$ and suppose

$$
\begin{aligned}
\log p(\boldsymbol{\theta} \mid \boldsymbol{Y})= & \log p\left(\boldsymbol{\theta}_{*} \mid \boldsymbol{Y}\right)+\Delta(\boldsymbol{\theta})^{T} F_{N}\left(\boldsymbol{\theta}_{*}\right) \\
& -0.5 \Delta(\boldsymbol{\theta})^{T} J_{N}\left(\boldsymbol{\theta}_{*}\right) \Delta(\boldsymbol{\theta})\left[1+o_{p}(1)\right]
\end{aligned}
$$

and

$$
\begin{aligned}
\log p\left(\boldsymbol{\theta} \mid \boldsymbol{Y}_{[S]}\right)= & \log p\left(\boldsymbol{\theta}_{*} \mid \boldsymbol{Y}_{[S]}\right)+\Delta(\boldsymbol{\theta})^{T} F_{N,[S]}\left(\boldsymbol{\theta}_{*}\right) \\
& -0.5 \Delta(\boldsymbol{\theta})^{T} J_{N,[S]}\left(\boldsymbol{\theta}_{*}\right) \Delta(\boldsymbol{\theta})\left[1+o_{p}(1)\right]
\end{aligned}
$$

uniformly for all $\boldsymbol{\theta} \in B\left(\boldsymbol{\theta}_{*}, \delta_{0} / \sqrt{N}\right)=\left\{\boldsymbol{\theta}: \sqrt{N}\left\|\boldsymbol{\theta}-\boldsymbol{\theta}_{*}\right\| \leq\right.$ $\left.\delta_{0}\right\}$. Moreover, $N^{-1 / 2} F_{N}\left(\boldsymbol{\theta}_{*}\right)=O_{p}(1), N^{-1 / 2} F_{N,[S]}\left(\boldsymbol{\theta}_{*}\right)=$ $O_{p}(1), \quad \max _{S \in I_{S}} \sup _{\boldsymbol{\theta}, \boldsymbol{\theta}^{\prime} \in B\left(\boldsymbol{\theta}_{*}, N^{-1 / 2} \delta_{0}\right)} \| J_{N,[S]}(\boldsymbol{\theta}) \quad-$ $J_{N,[S]}\left(\boldsymbol{\theta}^{\prime}\right) \|=o_{p}(N)$

$$
\begin{aligned}
0< & \inf _{\boldsymbol{\theta} \in B\left(\boldsymbol{\theta}_{*}, \delta_{0} N^{-1 / 2}\right)} \lambda_{\min }\left(n^{-1} J_{N}(\boldsymbol{\theta})\right) \\
& \leq \sup _{\boldsymbol{\theta} \in B\left(\boldsymbol{\theta}_{*}, \delta_{0} N^{-1 / 2}\right)} \lambda_{\max }\left(N^{-1} J_{N}(\boldsymbol{\theta})\right)<\infty
\end{aligned}
$$

and

$$
\begin{aligned}
0 & <\min _{S \in I_{S}} \inf _{\theta \in B\left(\boldsymbol{\theta}_{*}, \delta_{0} N^{-1 / 2}\right)} \lambda_{\min }\left(N^{-1} J_{N,[S]}(\boldsymbol{\theta})\right) \\
& \leq \max _{S \in I_{S}} \sup _{\boldsymbol{\theta} \in B\left(\boldsymbol{\theta}_{*}, \delta_{0} N^{-1 / 2}\right)} \lambda_{\max }\left(N^{-1} J_{N,[S]}(\boldsymbol{\theta})\right)<\infty .
\end{aligned}
$$

Assumption C3. Assume that for small $\delta_{0}>0$, if $N_{S} \leq N_{0}$, a fixed constant, then

$$
\max _{S \in I_{S}} \sup _{\boldsymbol{\theta} \in B\left(\boldsymbol{\theta}_{*}, \delta_{0}\right)}\left\|\partial_{\boldsymbol{\theta}} \log p_{S}(\boldsymbol{\theta})\right\|=O_{p}(1)
$$

and

$$
\max _{S \in I_{S}} \sup _{\boldsymbol{\theta} \in B\left(\boldsymbol{\theta}_{*}, \delta_{0}\right)}\left\|\partial_{\boldsymbol{\theta}}^{2} \log p_{S}(\boldsymbol{\theta})\right\|=o_{p}(N) .
$$

Assumption $C 4 \cdot \log p(\boldsymbol{\theta} \mid \boldsymbol{Y})$ and $\log p\left(\boldsymbol{\theta} \mid \boldsymbol{Y}_{[S]}\right)$ for all $S \in I_{S}$ are Laplace regular [19].

Assumption C5. $\lim _{N_{I_{S}} \rightarrow \infty} N_{B}^{-1} E\left[K_{N}\left(I_{S} \mid \boldsymbol{\theta}_{*}\right)\right]=K_{*}\left(I_{S}\right)$ and $\lim _{N \rightarrow \infty} N^{-1} E\left[J_{N}\left(\boldsymbol{\theta}_{*}\right)\right]=J_{*}$, where the expectation is taken with respect to the true data generator. Moreover, for a small $\delta_{0}>0$, we have

$$
\sup _{\boldsymbol{\theta} \in B\left(\boldsymbol{\theta}_{*}, \delta_{0}\right)}\left\|K_{N}\left(I_{S} \mid \boldsymbol{\theta}\right)-E\left[K_{N}\left(I_{S} \mid \boldsymbol{\theta}\right)\right]\right\|=o_{p}(1)
$$

and

$$
\sup _{\boldsymbol{\theta} \in B\left(\boldsymbol{\theta}_{*}, \delta_{0}\right)}\left\|J_{N}\left(I_{S} \mid \boldsymbol{\theta}\right)-E\left[J_{N}\left(I_{S} \mid \boldsymbol{\theta}\right)\right]\right\|=o_{p}(1) .
$$

Assumption C6. Each component of $N_{B}^{-1} \sqrt{N}\left\{K_{N}\left(I_{S} \mid \boldsymbol{\theta}_{*}\right)-\right.$ $\left.E\left[K_{N}\left(I_{S} \mid \boldsymbol{\theta}_{*}\right)\right]\right\}$ is asymptotically tight.

Remarks: Assumptions C1 and C2 are very general conditions and have been widely used to examine the asymptotic properties of the extremum estimator, such as the maximum likelihood estimate in general parametric models such as time series models [3]. Sufficient conditions of Assumptions $\mathrm{C} 1$ and $\mathrm{C} 2$ have been extensively discussed in the literature [3]. Assumption C3 is needed to examine the asymptotic properties of the three case influence measures for each $S \in I_{S}$. Most models with a smooth likelihood automatically satisfy Assumption C3. Assumption C4 is needed to use the Laplace approximation formula [19, 36]. Assumption C5 is ensured by the law of large numbers [38]. Assumption C6 is usually ensured by central limit theory. Recall 
that $p_{S}(\boldsymbol{\theta})=p\left(\boldsymbol{Y}_{S} \mid \boldsymbol{Y}_{[S]}, \boldsymbol{\theta}\right)$. If $p_{S}(\boldsymbol{\theta})$ only depends on a few observations in $\boldsymbol{Y}_{[S]}$, then we can apply the theory of U-statistics to establish Assumption C6 [37].

Proof of Theorem 1. It follows from Assumptions C1-C3 that we can expand $\log p_{S_{k}}\left(\tilde{\boldsymbol{\theta}}_{\left[S_{k}\right]}\right)$ at $\tilde{\boldsymbol{\theta}}$ for each $S$ and obtain

$$
\begin{aligned}
& \sum_{S_{k} \in I_{S}} \log p_{S_{k}}\left(\tilde{\boldsymbol{\theta}}_{\left[S_{k}\right]}\right) \\
= & \sum_{S_{k} \in I_{S}} \log p_{S_{k}}(\tilde{\boldsymbol{\theta}})+\sum_{S_{k} \in I_{S}} \partial_{\boldsymbol{\theta}} \log p_{S_{k}}(\tilde{\boldsymbol{\theta}})^{T} \Delta_{S_{k}}\left[1+o_{p}(1)\right],
\end{aligned}
$$

where $\Delta_{S_{k}}=\tilde{\boldsymbol{\theta}}_{\left[S_{k}\right]}-\tilde{\boldsymbol{\theta}}$. It follows from Proposition 1 (c) that

$$
\begin{aligned}
& \sum_{S_{k} \in I_{S}} \log p_{S_{k}}\left(\tilde{\boldsymbol{\theta}}_{\left[S_{k}\right]}\right) \\
= & \sum_{S_{k} \in I_{S}} \log p_{S_{k}}(\tilde{\boldsymbol{\theta}})-\sum_{S_{k} \in I_{S}}\left[\partial_{\boldsymbol{\theta}} \log p_{S_{k}}(\tilde{\boldsymbol{\theta}})\right]^{T}\left[J_{n}(\tilde{\boldsymbol{\theta}})\right]^{-1} \\
& \partial_{\boldsymbol{\theta}} \log p_{S_{k}}(\tilde{\boldsymbol{\theta}})\left[1+o_{p}(1)\right],
\end{aligned}
$$

which yields Theorem 1 (i). Theorem 1 (ii) directly follows from Assumptions C1, C2, and C5.

Proof of Theorem 2. We consider the exhaustive splitting for the leave-M clusters-out $\mathrm{CV}$. For any $S_{k}=$ $\left\{\left\{i_{1}\right\}, \ldots,\left\{i_{M}\right\}\right\}$, we have

$$
\begin{aligned}
& \log p_{S_{k}}\left(\tilde{\boldsymbol{\theta}}\left(M_{l}\right), M_{l}\right)=\sum_{l=1}^{M} \log p\left(\boldsymbol{Y}_{i_{l}} \mid \boldsymbol{\theta}, M_{l}\right), \\
& \sum_{S_{k} \in I_{S}} \log p_{S_{k}}\left(\tilde{\boldsymbol{\theta}}\left(M_{l}\right), M_{l}\right) \\
= & \left(\begin{array}{c}
n-1 \\
M-1
\end{array}\right) \sum_{i=1}^{n} \log p\left(\boldsymbol{Y}_{i} \mid \boldsymbol{\theta}, M_{l}\right),
\end{aligned}
$$

and

$$
\begin{aligned}
& \sum_{S_{k} \in I_{S}} E\left\{\left[\partial_{\boldsymbol{\theta}} \log p_{S_{k}}\left(\boldsymbol{\theta}_{*}\right)\right]^{\otimes 2}\right\} \\
= & \left(\begin{array}{c}
n-1 \\
M-1
\end{array}\right) \sum_{i=1}^{n} E\left\{\partial_{\boldsymbol{\theta}} \log p\left(\boldsymbol{Y}_{i} \mid \boldsymbol{\theta}_{*}\right)^{\otimes 2}\right\} .
\end{aligned}
$$

Therefore, we have

$$
\operatorname{BCIC}\left(I_{L M O}, M_{l}\right)=\left(\begin{array}{c}
n-1 \\
M-1
\end{array}\right) \operatorname{BCIC}\left(I_{L O O}, M_{l}\right)
$$

which yields Theorem 2 (i). Theorem 2 (ii) directly follows from Assumption C6.

Received 7 December 2013

\section{REFERENCES}

[1] Akaike, H., 1974. A New Look at the Statistical Model Identification. IEEE Transactions On Automatic Control 19, 716-723. MR0423716

[2] Ando, T., 2007. Bayesian predictive information criterion for the evaluation of hierarchical Bayesian and empirical bayes models. Biometrika 94, 443-458. MR2380571

[3] Andrews, D. W. K., 1999. Estimation when a parameter is on a boundary. Econometrica 67, 1341-1383. MR1720781

[4] Arlot, S., Celisse, A., 2010. A survey of cross-validation procedures for model selection. Statistics Surveys 4, 40-79. MR2602303

[5] Box, G. E. P., Tiao, G. C., 1968. A Bayesian approach to some outlier problems. Biometrika 55, 119-129. MR0225427

[6] Bunke, O., Milhaud, X., 1998. Asymptotic behavior of Bayes estimates under possibly incorrect models. The Annals of Statistics 26 (2), 617-644. MR1626075

[7] Chaloner, K., 1991. Bayesian residual analysis in the presence of censoring. Biometrika 78, 637-644. MR1130932

[8] Chen, M.-H., Dey, D. K., Ibrahim, J. G., 2004. Bayesian criterion based model assessment for categorical data. Biometrika 91 (1), 45-63. MR2050459

[9] Cook, R. D., 1977. Detection of influential observation in linear regression. Technometrics 19, 15-18. MR0436478

[10] Cook, R. D., Weisberg, S., 1982. Residuals and Influence in Regression. Chapman \& Hall Ltd. MR0675263

[11] CsiszÁr, I., 1967. Information-type measures of difference of probability distributions and indirect observations. Studia Scientiarum Mathematicarum Hungarica 2, 299-318. MR0219345

[12] Geisser, S., 1993. Predictive Inference: An Introduction. London: Chapman \& Hall Ltd. MR1252174

[13] Geisser, S., EdDy, W. F., 1979. A predictive approach to model selection (Corr: V75 P765). Journal of the American Statistical Association 74, 153-160. MR0529531

[14] Gelfand, A. E., Dey, D. K., Chang, H., 1992. Model Determination Using Predictive Distributions, with Implementation Via Sampling-based Methods (Disc: P160-167). In: Bernardo, J. M., Berger, J. O., Dawid, A. P., Smith, A. F. M. (Eds.), Bayesian Statistics 4. Oxford, Oxford University Press, pp. 147-159. MR1380275

[15] Gelfand, A. E., Ghosh, S. K., 1998. Model choice: A minimum posterior predictive loss approach. Biometrika 85, 1-11. MR1627258

[16] Ibrahim, J. G., Chen, M.-H., Sinha, D., 2001. Criterion-based methods for Bayesian model assessment. Statistica Sinica 11 (2) 419-443. MR1844533

[17] Ibrahim, J. G., Laud, P. W., 1994. A Predictive approach to the analysis of designed experiments. Journal of the American Statistical Association 89, 309-319. MR1266302

[18] Johnson, W., Geisser, S., 1983. A predictive view of the detection and characterization of influential observations in regression analysis. Journal of the American Statistical Association 78, 137144. MR0696858

[19] Kass, R. E., Kadane, J. B., Tierney, L., 1990. Asymptotic evaluation of integrals arising in Bayesian inference. In: Page, C., LePage, R. (Eds.), Computing Science and Statistics: Proceedings of the Symposium on the Interface. Springer-Verlag Inc, pp. 38-42.

[20] Kass, R. E., Tierney, L., Kadane, J. B., 1989. Approximate methods for assessing influence and sensitivity in Bayesian analysis. Biometrika 76, 663-674. MR1041411

[21] Konishi, S., Ando, T., Iмото, S., 2004. Bayesian information criteria and smoothing parameter selection in radial basis function networks. Biometrika 91, 27-43. MR2050458

[22] Konishi, S., Kitagawa, G., 1996. Generalised information criteria in model selection. Biometrika 83, 875-890. MR1440051

[23] Laud, P., Ibrahim, J., 1995. Predictive model selection. Journal of the Royal Statistical Society, Series B 57, 247-262. MR1325389 
[24] Lv, J., LiU, J. S., 2014. Model selection principles in misspecified models. J. R. Statist. Soc. B.

[25] McCulloch, R. E., 1989. Local model influence. Journal of the AmericanStatistical Association 84, 473-478.

[26] Merikangas, K. R., Stevens, D. E., Fenton, B., Stolar, M., O'Malley, S., Woods, S., Risch, N., 1998. Co-morbidity and familial aggregation of alcoholism and anxiety disorders. Psychological Medicine 28, 773-788.

[27] Murata, N., Yoshizawa, S., Amari, S., 1994. Network information criterion determining the number of hidden units for an artificial neural network model. IEEE Transactions on Neural Networks 5, 865-872.

[28] Peng, F., Dey, D. K., 1995. Bayesian analysis of outlier problems using divergence measures. The Canadian Journal of Statistics/La Revue Canadienne de Statistique 23, 199-213.

[29] Pettit, L. I., 1986. Diagnostics in Bayesian model choice. The Statistician: Journal of the Institute of Statisticians 35, 183-190.

[30] Ripley, B. D., 1996. Pattern Recognition and Neural Networks. Cambridge University Press. MR1438788

[31] Schwarz, G., 1978. Estimating the dimension of a model. The Annals of Statistics 6, 461-464. MR0468014

[32] Spiegelhalter, D. J., Best, N. G., Carlin, B. P., van der Linde, A., 2002. Bayesian measures of model complexity and fit (with discussion). Journal of the Royal Statistical Society, Series B: Statistical Methodology 64, 583-639. MR1979380

[33] Stone, M., 1974. Cross-validatory choice and assessment of statistical predictions (with discussion) (Corr: 76V38 P102). Journal of the Royal Statistical Society, Series B: Methodological 36, 111147. MR0356377

[34] Stone, M., 1977. An asymptotic equivalence of choice of model by cross-validation and Akaike's criterion. Journal of the Royal Statistical Society, Series B: Methodological 39, 44-47. MR0501454

[35] Takeuchi, K., 1976. Distribution of information statistics and critera for adequacy of models (in Japanese). Mathematical Sciences $153,12-18$.

[36] Tierney, L., Kass, R. E., Kadane, J. B., 1989. Fully exponential Laplace approximations to expectations and variances of nonpositive functions. Journal of the American Statistical Association 84, 710-716. MR1132586

[37] VAn der VAart, A. W., 1998. Asymptotic Statistics. Cambridge University Press. MR1652247

[38] van der VaArt, A. W., Wellner, J. A., 1996. Weak Convergence and Empirical Processes. Springer-Verlag Inc. MR1385671
[39] Vehtari, A., Ojanen, J., 2012. A survey of Bayesian predictive methods for model assessment, selection and comparison. Statistics Surveys 6, 142-228. MR3011074

[40] Zhang, H., 1999. Analysis of infant growth curves using multivariate adaptive splines. Biometrics 55, 452-459.

[41] Zhang, P., 1993. Model selection via multifold cross validation. The Annals of Statistics 21, 299-313. MR1212178

[42] Zhu, H., Ibrahim, J., Cho, N., Tang, N., 2010. A general review of Bayesian influence analysis, In: Frontier of statistical decision making and Bayesian analysis, Chen, M. H., Dey, D. K., Muller, P., Sun D. and Ye, K. (Eds.) New York, Springer-Verlag, pp. 219-237.

[43] Zhu, H., Ibrahim, J., Cho, N., Tang, N. S., 2012. Bayesian case-deletion measures for statistical models with missing data. Journal of Computational and Graphical Statistics 21, 253-271. MR2913366

[44] Zhu, H. T., Ibrahim, J. G., Cho, H. S., 2012. Perturbation and scaled cook's distance. Annals of Statistics 40, 785-811. MR2933666

Hongtu Zhu

Department of Biostatistics

University of North Carolina at Chapel Hill

Chapel Hill, NC, 27599

USA

E-mail address: hzhu@bios.unc.edu

Joseph G. Ibrahim

Department of Biostatistics

University of North Carolina at Chapel Hill

Chapel Hill, NC, 27599

USA

E-mail address: ibrahim@bios.unc.edu

Qingxia Chen

Department of Biostatistics

Vanderbilt University

Nashville, TN, 37232

USA

E-mail address: cindy. chen@vanderbilt.edu 